\title{
A 90-day Oral Exposure to Food-grade Gold at Relevant Human Doses Impacts the Gut Microbiota and the Local Immune System in a Sex-dependent Manner in Mice
}

\section{Lauris Evariste}

Toxalim UMR1331 (Research Centre in Food Toxicology), Toulouse University, INRAE, ENVT, INP-Purpan, UPS

\section{Bruno Lamas}

Toxalim UMR1331 (Research Centre in Food Toxicology), Toulouse University, INRAE, ENVT, INP-Purpan, UPS

\section{Sandrine Ellero-Simatos}

Toxalim UMR1331 (Research Centre in Food Toxicology), Toulouse University, INRAE, ENVT, INP-Purpan, UPS

\section{Laure Khoury}

PrediTox

\section{Christel Cartier}

Toxalim UMR1331 (Research Centre in Food Toxicology), Toulouse University, INRAE, ENVT, INP-Purpan, UPS

\section{Eric Gaultier}

Toxalim UMR1331 (Research Centre in Food Toxicology), Toulouse University, INRAE, ENVT, INP-Purpan, UPS

\section{Benoit Chassaing}

INSERM U1016, team "Mucosal microbiota in chronic inflammatory diseases", CNRS UMR 8104, Université de Paris

\section{Nicolas Feltin}

Department of materials, LNE

\section{Laurent Devoille}

Department of materials, LNE

\section{Georges Favre}

Department of materials, LNE

\section{Marc Audebert}

Toxalim UMR1331 (Research Centre in Food Toxicology), Toulouse University, INRAE, ENVT, INP-Purpan, UPS

Eric Houdeau ( $\nabla$ eric.houdeau@inrae.fr) 
Toxalim UMR1331 (Research Centre in Food Toxicology), Toulouse University, INRAE, ENVT, INP-Purpan, UPS

\section{Research Article}

Keywords: Edible gold, E175 food additive, nanoparticles, microbiota, intestinal immune response, sexual dimorphism

Posted Date: February 24th, 2022

DOI: https://doi.org/10.21203/rs.3.rs-1380222/v1

License: (c) (1) This work is licensed under a Creative Commons Attribution 4.0 International License. Read Full License 


\section{Abstract}

Background: Edible gold (Au) is commonly used as a food additive (E175 in EU) for confectionery and cake decorations, coatings and in beverages. Food-grade gold is most often composed of thin Au sheets or flakes exhibiting micro- and nanometric dimensions in their thickness. Concerns about the impact of mineral particles used as food additives on human health are increasing with respect to the particular physico-chemical properties of nanosized particles, which enable them to cross biological barriers and interact with various body cell compartments. In this study, male and female mice were exposed daily to E175 or an Au nanomaterial (Ref-Au) incorporated into food at relevant human dose for 90 days in order to determine the potential toxicity of edible gold.

Results: E175 or Ref-Au exposure in mice did not induce any histomorphological damage of the liver, spleen or intestine, nor any genotoxic effects in the colon and liver despite an apparent higher intestinal absorption level of Au particles in mice exposed to Ref-Au compared to the E175 food additive. While exposure to Ref-Au did no impacted intestinal microbiota, a marked alteration in the gut microbiota characterized by an increased Firmicutes/Bacteroidetes ratio and Proteobacteria abundance as well as a decreased production of short-chain fatty acids (SCFAs) was observed following E175 treatment of female mice compared to males. Moreover, increased production of IL-6, TNFa and IL-1 $\beta$ were observed in the colon of female mice at the end of the 90 -day exposure to E175, while in contrast, decreased IL-6, IL$1 \beta, \mathrm{IL}-17$ and TGF $\beta$ levels were found in the male colon.

Conclusions: These results revealed that a 90-day exposure to E175 added to the diet alters the gut microbiota and intestinal immune response in a sex-dependent manner in mice. Within the dose range of human exposure to E175, these alterations remained low in both sexes and mostly appeared to be nontoxic. However, at the higher dose, the observed gut dysbiosis and the intestinal low-grade inflammation in female mice could favour the occurrence of metabolic disorders supporting the establishment of toxic reference values for the safe use of gold as food additive.

\section{Introduction}

Gold (Au) is commonly used as a food additive (referred to as E175 in the EU) for the decoration of confectionery, cakes, chocolates and liquors. As with other food additives, such as E171 (titanium dioxide) or E174 (silver), food-grade Au is composed of micro- and/or nanosized particles and is representative of manufactured agents contributing to human daily exposure to nanoparticles through the diet [1-3]. The consumption of products containing E175 leads to a mean daily intake of Au reaching up to $0.31 \mu \mathrm{g} / \mathrm{kg}$ of body weight (BW)/day (d) in children and was estimated to possibly reach up to 1.32 $\mu \mathrm{g} / \mathrm{kg} \mathrm{BW} / \mathrm{d}$ across all population groups [4,5]. Despite the lack of relevant toxicological data, the consumption of E175 was considered acceptable in 1975 by the Scientific Community for Food, and no acceptable daily intake was determined [5,6]. There is a rising concern about the potential health risk associated with the presence of nanoparticles (NPs) in food products, as they could exert deleterious effects on health through the alteration of the intestinal flora, gut barrier integrity, and brain development 
and behaviour [7-10]. These effects are potentially related to the particular physicochemical properties of NPs, which enable them to cross biological barriers such as the intestine, blood-brain barrier and placenta [11-13]. The European Commission requested a scientific opinion on the re-evaluation of the use of edible gold (E175) as a food additive that pointed out the absence of data on E175 chronic toxicity [5]. Indeed, to date, the main studies have focused on in vitro toxicological effects associated with Au-NP exposure, indicating nonlinear size-dependent cytotoxicity [14], genotoxicity [15,16] or cellular uptake $[17,18]$. On the other hand, due to their potential use for therapeutic applications as nanocarriers [19], most of the in vivo studies conducted emphasized the biodistribution of the compounds, highlighting size-related biodistribution and excretion routes [20-25], with a strong influence of the surface characteristics and administration route [26-28]. Concerning the toxicological effects associated with in vivo exposure to Au-NPs, discrepancies between studies on rodents are noted, with responses ranging from no effect to weight loss, proinflammatory responses and granuloma formation in the liver or high mortality [28-31]. However, neither the nanomodels (100\% NPs) nor the administration methods used (e.g., intravenous or intraperitoneal injections) are representative of the food risk encountered by the general population, that is, chronic oral exposure at low doses to a mix of micro- and nanoparticles, as found in the E175 food additive. Indeed, studies of Au-NP toxicity should consider exposure to foodborne Au-NPs, i.e., E175-related, consumed daily by the population through the diet.

Following ingestion, Au-NPs are able to interact with bacteria from the intestinal microbiota [32] before crossing through the gut epithelial barrier $[28,32-34]$ to interact with local and systemic immune cells [35-37]. The intestinal mucosa represents a complex interface participating in the homeostatic relationship between the gut microbiota and host immune system in response to the environment and diet [38-40]. Indeed, the gut microbiota plays a key role in multiple physiological functions such as energy metabolism, gut immunity and brain development [40-43]. Reciprocally, intestinal epithelial cells and the immune system regulate and shape the gut microbiota $[39,40,44]$. The crosstalk between the microbiota and host cells is complex and involves bacterial metabolites, such as short-chain fatty acids (SCFAs) and aryl hydrocarbon receptor (AhR) ligands, which are essential for the maintenance of intestinal, nervous system and liver functions [45-48]. Many chronic diseases in humans are associated with alterations along the microbiota-immune system axis, such as inflammatory bowel disease and metabolic disorders [49]. Based on the observation that Au-NPs harbour antibacterial activities [50], chronic oral exposure to the corresponding food form (E175) could alter the gut microbiota, leading to dysbiosis with adverse health outcomes. Moreover, as sex-related differences have been noticed in the gut microbiota composition and immune responses [51-53], sexual dimorphism could exist with regard to the impacts of food-grade Au on the gut microbiota-immune systems axis, leading to a sex-related susceptibility to disease development. In this context, the aim of this study in mice was to explore the fate of the food additive E175 and to determine the potential physiological impact on the digestive tract (intestinal tolerance, including on the gut microbiota) of chronic exposure to E175 at relevant human doses, taking into account a potential sexual dimorphism. Particular attention was given to the size of the particles of the E175 test powder in comparison to a reference (Ref) Au test nanomaterial. 


\section{Results}

\section{Physicochemical characteristics of Au particles}

Scanning electron microscopy (SEM) and transmission electronic microscopy (TEM) coupled to energydispersive X-ray (EDX) analyses demonstrated that the E175 powder was composed of fragments of gold foils, which were close to purity (Fig. 1A, B). The mean thickness of the fragments of gold foils from E175 was $119 \pm 8.3 \mathrm{~nm}$ with a thickness size distribution ranging from 60 to $240 \mathrm{~nm}$, and $28 \%$ of the nanoobjects by number were named nanoplates because only one dimension was less than $100 \mathrm{~nm}$ (Fig. 1C). According to the manufacturer, the Au-NPs used in this study (Ref-Au) were spherical in shape, and the particle size distribution ranged from 50 to $100 \mathrm{~nm}$. The nanoparticles from Ref-Au were close to purity with a specific surface area of $3.5 \mathrm{~m}^{2} / \mathrm{g}$ (Fig. 1D, E). The nickel peak observed in the EDX spectra of E175 and Ref-Au was from the grid that supported the particles for the microscopy analysis (Fig. 1B, E).

\section{Animal behaviour, feed consumption and body and organ weights}

To determine the potential toxicity of food-grade Au (E175), male and female mice were exposed for 13 weeks to E175 incorporated into food pellets at relevant human dose levels of 0.1 and $1 \mu \mathrm{g} / \mathrm{kg} \mathrm{BW} / \mathrm{d}$ and at a high dose level of $10 \mu \mathrm{g} / \mathrm{kg} \mathrm{BW} / \mathrm{d}$. For comparison purposes of the potential size effect of $\mathrm{Au}$ particles, groups of male and female mice were treated with a 100\% nanosized Au nanomaterial (Ref-Au) incorporated into the food pellets at the higher dose level of $10 \mu \mathrm{g} / \mathrm{kg} \mathrm{BW} / \mathrm{d}$. Control male and female mice were fed an untreated diet. During the experiment, all mice appeared healthy, and no abnormal behaviour was observed in mice treated with E175 or Ref-Au. The daily feed intake of male and female mice exposed to E175 or Au-NPs was similar to that of the control group throughout the exposure period except at Day 3 after the beginning of the treatment, where the daily feed intake of male and female mice exposed to E175 at 0.1 and $1 \mu \mathrm{g} / \mathrm{kg} \mathrm{BW} / \mathrm{d}$ was significantly decreased compared to that of the control mice (Fig. 2A, B). A decrease in daily feed intake was also observed in female mice exposed to Ref-Au compared to the control group at Day 3 after the beginning of the exposure (Fig. 2B). These modulations in the daily feed intake observed only 3 days after the beginning of the treatment had no consequences on the body weights and body weight gain of male and female mice (Fig. 2C, D and Fig. S1A, B). Absolute liver and spleen weights were also similar between treated and control mice regardless of sex (ANOVA, $p$ $>0.05$ ) (Table 1). The mean exposure doses to E175 and Ref-Au were determined using the recorded pellet consumption and body weights. The feed effective intake was $90 \%$ and $110 \%$ of the target intake for male and female mice, respectively, showing that the mean exposure doses to E175 and Ref-Au were very close to the target exposure doses (Table S1). 
Table 1

Body and organ weights in mice exposed to Ref-Au or E175 for 13 weeks.

\begin{tabular}{|c|c|c|c|c|c|}
\hline Sex & Group & $\begin{array}{l}\text { Body weight } \\
\text { at sacrifice (g) }\end{array}$ & $\begin{array}{l}\text { Body weight gain } \\
\text { at sacrifice (\%) }\end{array}$ & Liver (g) & Spleen (mg) \\
\hline \multirow[t]{9}{*}{ Male } & Control & $31.7 \pm 0.8$ & $49.7 \pm 2.4$ & $1.43 \pm 0.04$ & $81.16 \pm 3.50$ \\
\hline & Ref-Au & $31.9 \pm 0.7$ & $52.2 \pm 2.8$ & $1.48 \pm 0.05$ & $95.55 \pm 6.81$ \\
\hline & $10 \mu \mathrm{g} / \mathrm{kg} \mathrm{BW} / \mathrm{d}$ & & & & \\
\hline & E175 & $30.5 \pm 0.7$ & $44.7 \pm 2.5$ & $1.40 \pm 0.06$ & $78.00 \pm 3.08$ \\
\hline & $0.1 \mu \mathrm{g} / \mathrm{kg} \mathrm{BW} / \mathrm{d}$ & & & & \\
\hline & E175 & $29.3 \pm 0.6$ & $44.6 \pm 1.9$ & $1.37 \pm 0.05$ & $75.84 \pm 1.63$ \\
\hline & $1 \mu \mathrm{g} / \mathrm{kg} \mathrm{BW} / \mathrm{d}$ & & & & \\
\hline & E175 & $30.9 \pm 0.8$ & $47.8 \pm 3.1$ & $1.38 \pm 0.04$ & $74.50 \pm 2.51$ \\
\hline & $10 \mu \mathrm{g} / \mathrm{kg} \mathrm{BW} / \mathrm{d}$ & & & & \\
\hline \multirow[t]{9}{*}{ Female } & Control & $22.7 \pm 0.3$ & $38.3 \pm 1.6$ & $1.03 \pm 0.02$ & $84.18 \pm 3.55$ \\
\hline & Ref-Au & $23.1 \pm 0.2$ & $41.2 \pm 1.6$ & $1.06 \pm 0.02$ & $81.60 \pm 2.91$ \\
\hline & $10 \mu \mathrm{g} / \mathrm{kg} \mathrm{BW} / \mathrm{d}$ & & & & \\
\hline & E175 & $22.3 \pm 0.2$ & $38.4 \pm 2.4$ & $1.04 \pm 0.04$ & $81.91 \pm 4.50$ \\
\hline & $0.1 \mu \mathrm{g} / \mathrm{kg} \mathrm{BW} / \mathrm{d}$ & & & & \\
\hline & E175 & $22.2 \pm 0.2$ & $34.5 \pm 2$ & $1.05 \pm 0.02$ & $76.58 \pm 2.81$ \\
\hline & $1 \mu \mathrm{g} / \mathrm{kg} \mathrm{BW} / \mathrm{d}$ & & & & \\
\hline & E175 & $22.2 \pm 0.3$ & $32.5 \pm 1.8$ & $1.01 \pm 0.02$ & $82.47 \pm 2.22$ \\
\hline & $10 \mu \mathrm{g} / \mathrm{kg} \mathrm{BW} / \mathrm{d}$ & & & & \\
\hline
\end{tabular}

\section{Gold tissue distribution}

Confocal microscopy for metal particle visualization associated with TEM-EDX analysis was used to monitor Au distribution in the digestive tract, liver and spleen of control mice or mice exposed for 13 weeks to Ref-Au and the highest dose of E175 $(10 \mu \mathrm{g} / \mathrm{kg} \mathrm{BW} / \mathrm{d})$. While almost no particles were detected in the digestive tract of control mice, the presence of laser-reflecting metal particles was observed in the lamina propria of the jejunum villi, in the nuclei of immune cells from the Peyer's patches and in the colonic lumen from mice fed daily with food containing the Ref-Au nanomaterial (Fig. S2-4). On TEM 
tissue sections from mice exposed to Ref-Au, inorganic particulate matter was also observed in the intestinal lumen, in the microvilli and the cytoplasm of jejunum enterocytes as well as in the microvilli of $M$ cells lining the dome of the Peyer's patch and in colonic epithelial cells (enterocytes and mucusproducing goblet cells) (Fig. S5-7). Particles were also recovered in areas lining blood vessels in the colonic section (Fig. S7). Chemical elemental mapping using TEM-EDX confirmed the presence of Au on particle deposits observed in the digestive tract of mice treated with Ref-Au (Fig. 3A-C).

In E175-exposed mice $(10 \mu \mathrm{g} / \mathrm{kg} \mathrm{BW} / \mathrm{d})$, some laser-reflecting metal particles were observed in the lumen of the jejunum and in the nuclei of immune cells from Peyer's patches, while no or rare particles were observed in the lumen of the colon (Fig. S2-4). TEM analysis of tissue sections from mice exposed to E175 showed that electron-dense inorganic particles were found in the intestinal lumen, in the microvilli and in the cytoplasm of jejunum enterocytes as well as close to the nucleus of immune cells in Peyer's patches and in the nucleus of cells located in the colonic lamina propria (Fig. S8-10). The presence of Au among the particles observed by TEM was confirmed in jejunum and colon tissue sections (Fig. 3D, E). No Au signal was detected in the analysed particles in Peyer's patches (Fig. S9). The particles recovered in the Peyer's patches of mice exposed to E175 were principally composed of silicon (Si) and/or copper (Cu) (Fig. S9). Interestingly, among the particles analysed by EDX in the digestive tract of mice exposed to Ref-Au or E175, Si- and titanium (Ti)-containing particles were frequently found in addition to Au (Fig. 3D and Fig. S9).

Gold distribution was also measured in the liver and spleen to determine whether Au particles from the diet containing the Ref-Au nanomaterial or the food-grade gold (E175) reached the systemic circulation and accumulated in these organs. No or rare metal particles were observed in the liver of control and E175-treated mice, while some laser-reflecting metal particles were recovered in the portal space close to the hepatic interlobular artery of mice treated with the Ref-Au nanomaterial (Fig. S11). Moreover, TEMEDX analysis confirmed the presence of Au on the particle deposits observed in the liver of mice treated with Ref-Au (Fig. 3F and Fig. S12). No Au signal was observed in the analysed particles in the liver of mice exposed to E175 (Fig. S13). The few electron-dense inorganic particles observed in the cytoplasm of hepatocytes of mice exposed to E175 were composed of Cu and calcium (Ca) (Fig. S13).

In the spleen of control mice, no or few laser-reflecting metal particles were found, while some fluorescent metal particles were observed in the vascular lumen of the splenic artery or translocated inside the splenic tissue of Ref-Au- and E175-treated mice (Fig. S14). TEM-EDX analysis revealed the presence of few electron-dense inorganic particles with Au signals in the cytoplasm of immune cells in the spleen from Ref-Au-treated mice, while no Au signal was detected in the rare electron-dense inorganic particles found in the spleen of mice exposed to E175 (Fig. 3 G and Fig. S15, 16).

Taken together, these results showed that Au particles translocated in the small and large intestine following 13 weeks of exposure to the Ref-Au nanomaterial or E175. The presence of Au particles in the liver and spleen emphasized a systemic passage of Au nanoparticles from the gut in mice treated with Ref-Au. In contrast, no evidence of systemic passage of Au particles was observed in mice exposed for 13 
weeks to a dose of E175 exceeding by 10-fold the high exposure level for humans in the maximum exposure level assessment scenario in the EFSA Scientific Opinion of gold (E175) as a food additive.

\section{Histopathology, genotoxicity and intestinal permeability}

Since Au particles were found in the small and large intestines of mice exposed to E175 and Ref-Au as well as in the liver and spleen of mice treated with Ref-Au, histopathological changes in H\&E-stained sections, genotoxicity and intestinal permeability were evaluated to determine potential alterations in these organs. No treatment-related histopathological lesions were identified in the jejunum, colon, liver or spleen of Ref-Au- and E175-exposed animals compared to control mice (Fig. 4A-E). Moreover, the 90-day oral exposure to Ref-Au and E175 did not induce increased phosphorylation of $\mathrm{YH} 2 \mathrm{AX}$, a biomarker of genotoxicity, in the colon and liver of male and female mice (Fig. 5A-D). Likewise, no increase in intestinal permeability nor alteration of epithelial integrity at the end of the Ref-Au or E175 treatment were observed in male and female mice (Fig. 5E-L). Slightly and significantly decreased lumen-to-mucosal permeability of FITC-dextran was noted in the ileum of males treated with E175 at $1 \mu \mathrm{g} / \mathrm{kg} \mathrm{BW} / \mathrm{d}$ compared to the control group (Fig. 5E). This decrease in the dextran flux was concomitant with an unmodified TER value (Fig. 5F). In addition, the decreased epithelial permeability to FITC-dextran did not occur in a dose-related pattern (similar trend at the high dose level but not significant). These slight changes may be considered to be related to the E175 treatment, but in view of the absence of a dose response (dextran flux and TER) and considering the decreased basal permeability rather than an increase, this effect was not considered deleterious. Altogether, these data demonstrate that the exposure to Ref-Au or E175 do not impair intestinal barrier integrity. In addition, the systemic distribution of gold nanoparticles previously observed is not associated to genotoxic effects in the liver.

\section{Faecal microbiota composition and function}

The systemic absorption of Au-NPs was low in rodents [33,34] and indicated that the majority of the ingested Au-NPs accumulate in the lumen of the gut, favouring permanent contact of the particles with the intestinal bacteria. In addition, based on the observation that Au-NPs and associated byproducts harbour antibacterial activities [50], chronic oral exposure to Au-NPs or their corresponding food forms could alter the gut microbiota, leading to dysbiosis. Therefore, the impact of subchronic oral exposure to Ref-Au or E175 on faecal microbiota composition and activity was explored.

Principal coordinate analysis revealed a difference in faecal microbiota profiles between male and female mice, regardless of the experimental condition considered (Fig. S17A). Based on this observation of a sexual dimorphism in intestinal microbiota composition in adult mice, subsequent analysis were conducted on each sex independently.

Exposure to Ref-Au did not affect the richness of the gut bacterial communities in male mice, while a significant increase was observed in female mice following exposure to the higher dose of E175 (Fig. 6A, $\mathrm{H})$. In addition, beta diversity analysis in female and male mice using Bray-Curtis distances indicated a 
significant increase of dissimilarities in bacterial community abundances between the control and the exposed groups regardless of the dose of the treatment (Fig. 6B, l; Fig. S17B, C).

The faecal microbiota of control and exposed male and female mice was dominated by members of the phyla Firmicutes, Bacteroidetes and Proteobacteria (Fig. S17D, E). No treatment-related gut microbiota alterations were identified at the phylum or genus levels in exposed male mice (Fig. 6C-G). In female mice, no differences in faecal microbiota composition were observed at the phylum level following exposure to Ref-Au, while the phyla Bacteroidetes and Actinobacteria were significantly decreased after exposure to the highest dose of E175, benefiting to the phyla Firmicutes and Proteobacteria (Fig. 6J-M). As a consequence, a significant increase in the Firmicutes/Bacteroidetes (F/B) ratio was observed following exposure to E175 at $10 \mu \mathrm{g} / \mathrm{kg}$ BW/d (Fig. 6N). Moreover, the abundance of Bifidobacterium (genus) was significantly decreased in female mice exposed to E175 at 1 and $10 \mu \mathrm{g} / \mathrm{kg} \mathrm{BW} / \mathrm{d}$, and the abundance of Akkermansia muciniphila (species) was increased after exposure to Ref-Au and E175 at 1 and $10 \mu \mathrm{g} / \mathrm{kg}$ BW/d (Fig. 60, P).

To gain insight into the functional differences between the intestinal microbiota from exposed and unexposed male and female mice, we explored the metabolic activity of the faecal microbiota using ${ }^{1} \mathrm{H}-$ NMR analysis. This approach allows the detection of 40-50 metabolites, most of which are either produced by gut microbiota metabolism or host-gut microbiota co-metabolism.

The ${ }^{1} \mathrm{H}$-NMR-based metabolomics showed that a 90-day exposure to Ref-Au induced significant changes in the faecal metabolic profiles in male (Fig. S18A-B) but not female mice (Fig. S18C-D). An orthogonal projection on latent structure-discriminant analysis (O-PLS-DA) significantly discriminated males exposed to Ref-Au from control males (O-PLS-DA model: $Q^{2} Y=0.42$ ). The metabolic profiles of the Ref-Au nanomaterial-treated male mice were mainly differentiated from the control group by an increased production level of aspartate (Fig. 7G and Fig. S18B).

Following 90 days of exposure to $\mathrm{E} 175$ at $0.1 \mu \mathrm{g} / \mathrm{kg} \mathrm{BW} / \mathrm{d}$, no significant difference relative to the control group was determined for either sex with the O-PLS-DA statistical model (O-PLS-DA model: $Q^{2} Y=0.14$ ) (Fig. 7A and Fig. 8A, D). However, the analysis of discriminant metabolites revealed higher levels of aspartate in male mice treated with the low dose of E175 compared to the control group (Fig. 7D, G). In addition, a clear discrimination between the metabolic profiles of the control group compared to male and female mice exposed to 1 and $10 \mu \mathrm{g} / \mathrm{kg}$ BW/d of E175 was observed (Fig. 7B, C and Fig. 8B, C).

Compared to the control, males exposed to $1 \mu \mathrm{g} / \mathrm{kg}$ BW/d E175 exhibited higher faecal levels of aspartate and medium-chain fatty acids (MCFAs) and lower levels of acetate and propionate, which are both shortchain fatty acids (SCFAs) (Fig. 7E, G-J). Analysis of discriminant metabolites in males exposed to 10 $\mu \mathrm{g} / \mathrm{kg} \mathrm{BW} / \mathrm{d}$ E175 showed higher levels of aspartate and lower levels of acetate and MCFAs than those in unexposed mice (Fig. 7F, G-J). In female mice exposed to 1 and $10 \mu \mathrm{g} / \mathrm{kg}$ BW/d E175, the metabolic profiles were differentiated from control mice mainly by lower levels of acetate and propionate (Fig. 8B, C, E-I). An increased level of MCFAs was also observed in females exposed to $1 \mu \mathrm{g} / \mathrm{kg} \mathrm{BW} / \mathrm{d}$ E175 compared to untreated controls (Fig. 8I). 
Altogether, these results demonstrate that subchronic exposure to Ref-Au is not sufficient to induce significant alterations of the faecal microbial composition in males and females, suggesting a limited effect of the nanosized Au particles at a high dose level. In contrast, the intestinal microbiota composition and production of metabolites involved in host physiology, such as SCFAs, were altered in male and female mice exposed to E175. These microbiota alterations induced by E175 ingestion were more pronounced in female mice, with a negative shift in the microbial community, favouring the growth of pathogenic bacteria such as Proteobacteria at the expense of beneficial strains such as Bifidobacterium.

\section{Intestinal immune response}

Our results presented above highlight that E175 subchronic treatment at a human-relevant dose decreased the faecal microbiota production of SCFAs, which play a role in intestinal immunity [54]. The presence of Au particles in the intestinal tissues of mice exposed to Ref-Au or E175 could suggested that Au particles could interact with intestinal immune cells. To determine whether daily exposure to Ref-Au or E175 impacts the intestinal immune response, we assessed the expression and production of pro- and anti-inflammatory cytokines in the colon of mice. Faecal lipocalin ( $L c n)-2$ levels, which is used as a general gut inflammatory marker [55], were also evaluated.

Compared to the level in the control group, a dose-dependent trend towards an increase in faecal Lcn-2 levels was noticed in female mice exposed to E175 that did not reach significance (ANOVA, p >0.05) (Fig. S19A). Similarly, no differences in faecal Lcn-2 levels were observed in male mice regardless of the treatment considered (Fig. S19B).

Most notably, decreased production of the proinflammatory cytokines IL-1 $1 \beta$, IL- 6 and IL-17 was observed in the colon of male mice exposed to all doses of E175 (Fig. 9A, B, E). This decrease in IL-1 $\beta$ levels followed a dose-related pattern, which was not reported for IL-6 and IL-17 (Fig. 9A, B, E). Accordingly, a significant downregulation of the gene expression of IL-1 $\beta$ was noted in male mice exposed to E175 (Fig. S20A). In contrast, no differences were observed for IL-17 gene expression or for TNFa expression and secretion, regardless of the E175 dose level (Fig. 9C and Fig. S20B, D). In male mice, the colonic production of the proinflammatory cytokine IFNY was significantly increased at a dose of $0.1 \mu \mathrm{g} / \mathrm{kg} \mathrm{BW} / \mathrm{d}$ E175 without a change in the corresponding gene expression (Fig. 9D and Fig. S20C). A 90-day exposure to E175 also affected the anti-inflammatory response in the colon of male mice through decreased production of the cytokine TGF $\beta$ following exposure to the food additive at 1 and $10 \mu \mathrm{g} / \mathrm{kg} \mathrm{BW} / \mathrm{d}$ (Fig. 9F). No differences occurred for TGF $\beta$ gene expression or for the expression and production of the anti-inflammatory cytokine IL-10 regardless of the dose of E175 (Fig. 9G and Fig. S20E, F). Concerning the impact of a 90-day exposure to Ref-Au in male mice, a significant decrease in IL-1 $\beta$ and IL-10 expression was observed without an effect on protein production (Fig. 9A, G and Fig. S20A, F). Moreover, decreased production of the cytokine TGF $\beta$ was reported in the colon of male mice treated with Ref-Au, while the gene expression of this anti-inflammatory mediator was unchanged (Fig. 9F and Fig. S20E). 
In female mice, a 90-day exposure to Ref-Au led to decreased colonic production of the proinflammatory cytokine TNFa (Fig. 9J). Although a similar decreasing trend was also observed for the gene expression of TNFa in the colon mucosa of female mice exposed to Ref-Au, this effect was not significant (Fig. $\mathrm{S} 2 \mathrm{OH}$ ). These findings were paralleled by higher IL-6 and TNFa secretion in the colons of female mice exposed to $1 \mu \mathrm{g} / \mathrm{kg}$ BW/d E175 without a change in TNFa expression compared to those of control mice (Fig. 9l, $\mathrm{J}$ and Fig. S2OH). In addition, an increase in the secretion of the proinflammatory cytokine IL-1 $\beta$ was reported in female mice exposed to 0.1 and $1 \mu \mathrm{g} / \mathrm{kg} \mathrm{BW} / \mathrm{d}$ E175, while the IL-1 $\beta$ level did not differ from that of the control group at the highest dose of E175 (Fig. 9H and Fig. S20G). The gene expression of IL-1 $\beta$ in the colon of female mice followed a dose-related pattern similar to that observed at the protein level, but the increased expression of this proinflammatory cytokine was statistically significant only at the intermediate dose of E175 (Fig. S20G). Gene expression of the proinflammatory cytokine IL-17 was also significantly increased in female mice exposed to $1 \mu \mathrm{g} / \mathrm{kg}$ BW/d E175 compared to control mice, but there was no change in IL-17 secretion (Fig. 9L and Fig. S20J). Last, the gene expression and protein production of the anti-inflammatory cytokines IL-10 and TGF $\beta$ in the colon mucosa of female mice exposed to E175 were not significantly different from those of control mice (Fig. 9M, N and Fig. S20K, L).

Altogether, these results showed that 90-day oral exposure to Ref-Au or E175 modulated the intestinal immune response in a sexually dimorphic manner. In male mice, except for an increased IFNy level only reported at the lowest dose of E175, the production of the proinflammatory cytokines IL-6, IL-1 $\beta$ and IL-17 as well as of the anti-inflammatory cytokine TGF $\beta$ was decreased at the end of E175 treatment. In contrast, similar oral exposure to E175 in female mice induced increased production of the proinflammatory cytokines IL-6, TNFa and IL-1 $\beta$. Moreover, oral exposure to Ref-Au induced a decreased production of TNFa only in female mice. These alterations in the intestinal immune response observed in both sexes were potentially due to the interaction of the Au particles with intestinal microbiota and/or intestinal immune cells.

\section{Effects of E175 exposure on intestinal AhR activity}

Our results showed that a 90-day exposure to E175 induced decreased production of IL-17 in the colon mucosa of male mice. One mechanism that could link these findings is the modulation of the aryl hydrocarbon receptor (AhR) by the microbiota within the gastrointestinal tract. Indeed, indole derivates generated through the metabolism of tryptophan by the intestinal microbiota have a role in the mucosal immune response via AhR by modulating immune cells that produce IL-17 [56,57]. Therefore, by examining the activation of AhR by the faecal microbiota, we are able to evaluate the capacity of the microbiota to induce IL-17 production by intestinal immune cells. Using an AhR reporter system, we found that faeces from male mice exposed to E175 were defective in their ability to activate AhR (Fig. 10A). In accordance with the absence of IL-17 modulation observed in the colon mucosa of female mice after E175 or Ref-Au exposure, the capacity of the faecal microbiota to activate AhR was not different in treated female mice compared to the control group (Fig. 10B). 
Collectively, these results showed that the decreased production of IL-17 observed in the colon of male mice exposed to E175 could be partly related to the reduced capacity of the faecal microbiota to produce derivates able to activate AhR. This defect in AhR activation by the intestinal microbiota was specifically observed in male mice and confirmed the sexual dimorphism noted in the intestinal production of IL-17 after oral exposure to E175.

\section{Discussion}

Edible gold ( $\mathrm{Au}$ ) is composed of mixed nano- and micron-sized thin sheets or flakes of elemental $\mathrm{Au}$ [58]. When manufactured as a food additive (referred to as E175 in the EU), gold is used in the human diet for food decoration, e.g., in cakes, chocolates, and ice cream, among other foodstuffs and drinks such as liqueurs [5]. Although recent reports based on nanoparticle models showed that nanosized Au can pass through the intestinal epithelium, no in vivo study has been carried out to investigate the tissue distribution of E175 particles along the gut and whether the nano fraction of the food additive presents a specific risk for consumers. Overall, a re-evaluation of edible $\mathrm{Au}$ as a food additive was requested by the European Commission, which pointed out the absence of data on E175 chronic toxicity [5]. In this context and based on the current scenario for dietary exposure in the general population [5], the present study in mice has investigated the intestinal absorption and the potential toxicity of the E175 food additive following exposure to relevant doses for humans. Special attention was given to the particle size-related effects in comparison to a pure Au nanomaterial (herein, Ref-Au, i.e., 100\% composed of NPs). To ensure relevance for risk assessment in humans [59], food-grade gold (E175) particles and Ref-Au were incorporated into a solid food matrix (rodent pellets), and mice were exposed for 13 weeks through the diet. No mortality was recorded, and body weight evolution appeared to be consistent and similarly increased in all groups, indicating no overt toxicity of the Au-NPs or the E175 additive at the tested doses. In addition, no treatment-related histomorphological damage in the liver, spleen and intestine or genotoxic effects in the colon and liver were observed for either compound at the end of the 13-week oral toxicity study. To date, the few studies on the oral toxicity of Au particles have been carried out in rats or mice using high concentrations of coated or uncoated Au-NPs (20 to 17,000 $\mu \mathrm{g} / \mathrm{kg} \mathrm{BW/d}$ ) administered in water suspension by gavage for shorter time periods (between 1 and 28 days) [28,32-34,60,61]. Contradictory results were reported, with some studies showing no or low toxicity based on body and organ weights as well as on histopathological, haematological and serum biochemical analysis [28,32$34,60]$, while others revealed deeper alterations in some of these parameters [28,36,61]. Differences in animal species, duration of exposure, dose, size, shape and coating of Au-NPs may contribute to these discrepancies. Furthermore, while in vivo studies in rodents concluded that oral Au-NPs entered the bloodstream and accumulated in the kidney and liver [28,32-34], these conditions using high dose levels and spherical nanomodels are not representative of E175 exposure in humans, which occurs at very low doses of ingested thin foils or larger sheets, some of which have dimensions $<100 \mathrm{~nm}$ in thickness (i.e., nanoplates).

In our study, after thirteen weeks of oral exposure to E175 added to food pellets, Au particles were commonly recovered in the lumen of the jejunum and colon of treated mice, whereas no Au element was 
evidenced in the liver and spleen. This observation highlighted the almost absent gut-to-blood passage of gold from the food additive, a situation that contrasted with Au-NP treatment herein recovered in the liver and spleen of mice exposed to the Ref-Au. Gold particles were shown to cross the intestinal epithelia in vitro with a size-dependent diffusion capacity, highlighting a higher permeability to Au-NPs than to their microparticulate forms [62]. Accordingly, our in vivo study confirmed the systemic passage of Au-NPs in mice exposed to pure nanosized matter, while no similar translocation was observed in mice exposed to the food additive E175, which was mainly composed of larger Au particles. It is reasonable to conclude that the physical form of edible gold in our batch of E175, where only $28 \%$ of the particles were on the order of nanosized in thickness, considerably limits their absorption along the gut. Of note, no significant change in intestinal permeability or food intake was observed in the Ref-Au group of mice, indicating that NP absorption did not result from a loss of epithelial barrier integrity or higher gold consumption during the treatment. On the other hand, the relatively low occurrence of Au signals in the liver of mice exposed to the Ref-Au suggested a limited hepatic retention of NPs after daily exposure for 90 days through food. This is in accordance with a previous biodistribution study of Au-NP models in rats, demonstrating low systemic absorption with elemental gold mainly eliminated through the faeces [34].

Due to the well-known antibacterial properties of elemental gold [50], it is conceivable that $\mathrm{Au}$ accumulation in the lumen of the digestive tract after long-term exposure to E175 could alter the intestinal microbiota, both in its microbial composition and metabolic activity. The gut microbiota plays a crucial role in several vital functions, such as digestive, metabolic and immune functions [49], but this important component of the gastrointestinal tract has received no attention after exposure to edible gold on a chronic basis. In our study, a principal coordinates analysis performed on the overall data indicated that the bacterial community composition significantly differed between male and female mice, regardless of the treatment condition, i.e., including in the untreated (control) groups. This result is in full accordance with previous studies highlighting a sex difference in microbiota composition [51,52,63], and this led us to suggest that the impact of Au particles on intestinal microbiota could differ between the sexes. Indeed, we reported more significant changes in the intestinal microbiota of female mice exposed to the Ref-Au or the food-grade Au form than in males. In female mice exposed for 13 weeks to the Ref$\mathrm{Au}$, the analysis of the richness and diversity of the bacterial communities in the intestine showed increased diversity in bacterial species with an increased abundance of Akkermansia muciniphila. The mucin-degrading bacterium Akkermansia muciniphila is recognized as beneficial through local antiinflammatory effects and has been shown to protect against metabolic disorders such as obesity [6466]. In a study using male mice exposed orally to a 1700-fold higher dose of Au-NPs, a decreased bacterial richness of the gut microbiota was observed together with an increased abundance of Firmicutes (mainly Enterococcus, Turibacter and Lachnospirace) and a decreased proportion of Bacteroidetes, such as Bacteroides, Alistipes and Rickenella [32]. Interestingly, some of these alterations in the gut microbiota were lost at lower doses of exposure as well as after coating the Au-NPs with 4,6diamino-2-pyrimidinethiol, showing a coating- and a dose-related effect of the Au-NPs on the gut microbiota [32]. In accordance, we observed a dose-related effect in our study, with more significant changes in the intestinal microbiota in females exposed to the highest dose of E175 $(10 \mu \mathrm{g} / \mathrm{kg} \mathrm{BW} / \mathrm{d})$. At 
this dosage and only in females, decreased proportions of Actinobacteria and Bacteroidetes together with increased frequencies of Firmicutes and Proteobacteria led to a significant increase in the F/B ratio. The production of the SCFAs acetate and propionate was also decreased at this dose level in this sex, while at $1 \mu \mathrm{g} / \mathrm{kg} \mathrm{BW} / \mathrm{d}$ E175, only the decreased abundance of Actinobacteria and the drop in SCFA levels remained significant. Changes in acetate and propionate levels could be linked to the decrease in Bifidobacterium, which belongs to the SCFA-producing phylum Actinobacteria [67]. None of these effects were reported at $0.1 \mu \mathrm{g} / \mathrm{kg} \mathrm{BW} / \mathrm{d}$ of E175, highlighting the dose-related changes in intestinal microbiota composition and activity in female mice. In chronic human diseases associated with gut dysbiosis, such as obesity and overweight [68,69], an altered F/B ratio is commonly reported [70-72] and helps to predict a decrease in the relative abundance of SCFAs $[73,74]$. Furthermore, Proteobacteria are often overrepresented in several intestinal and extraintestinal diseases with an inflammatory phenotype $[75,76]$. Similar F/B ratio and Proteobacteria changes were reported in female mice exposed to E175, suggesting that chronic exposure to high doses of edible gold could increase susceptibility to metabolic disorders. From a toxicological point of view, this emphasizes that the negative and dose-dependent shift linked to E175 exposure on the microbial community in the intestine can be considered a deleterious effect of the food additive. Most of these effects were first reported at a dose $(1 \mu \mathrm{g} / \mathrm{kg} \mathrm{BW} / \mathrm{d})$ close to dietary levels in humans according to the EFSA Opinion [5], with changes in the F/B ratio and proteobacteria abundance observed at 10 -fold above the high exposure level in these dietary scenarios. Interestingly, because only minor alterations in the intestinal microbiota occurred in the Ref-Au group of mice, regardless of sex, one may suggest that Au-NPs have very limited effects on the gut microbiome in contrast to larger Au particles in the E175 food additive.

Numerous studies have highlighted the existence of anti- or proinflammatory microorganisms in the gut. Indeed, metabolites derived from commensal bacteria (such as MCFAs, SCFAs and AhR ligands) can regulate immune cell functions via indirect as well as direct mechanisms $[39,40,49,77,78]$. For example, in the present report, the production defect of MCFAs and SCFAs by intestinal bacteria after 13 weeks of exposure to E175 suggested that local immune functions could be affected in response to E175-evoked gut dysbiosis. On the other hand, the biodistribution analysis of Au-NPs in mice exposed to the Ref-Au also showed that particles were recovered inside the Peyer's patches along the small intestine, suggesting direct interaction with immune cells. In our study, the inflammatory status of the intestine was first assessed using the faecal levels of Lcn-2, which is a protein mainly secreted in the intestinal lumen by neutrophils that provides a sensitive and broadly dynamic means to noninvasively detect intestinal inflammation in mice [55]. Because faecal Lcn-2 levels did not increase at the end of the 90-day treatment with the gold compounds, we reported that neither the Ref-Au nor the food form E175 at the tested doses induced severe inflammation in the gut in both male and female mice. A deeper analysis of the mucosal immune response, however, revealed decreased production of TNFa or TGF $\beta$ in the colon exposed to 10 $\mu \mathrm{g} / \mathrm{kg} \mathrm{BW} / \mathrm{d}$ of Ref-Au regardless of sex. No other significant changes in pro- or anti-inflammatory cytokines have been reported with Ref-Au, suggesting a limited effect of Au-NPs on the immune profile in the intestine in both sexes. Previous studies in male mice indicated that Au-NPs (of similar size to the one used in our study) induced spleen inflammation 7 days after intraperitoneal injection, and smaller 
particles administered at high doses by oral gavage or intravenously increased reactive oxygen species production and promoted inflammation in the liver or spleen [35-37]. Thus, the impact of Au-NPs on the immune response cannot be fully excluded depending on the dose, the administration route and the target organ considered.

Regarding food additives, no studies have evaluated the effects of chronic ingestion of E175 on the intestinal immune response. We demonstrated that long-term oral exposure to E175 at a human-relevant dose $(1 \mu \mathrm{g} / \mathrm{kg} \mathrm{BW} / \mathrm{d})$ induced increased production of the proinflammatory cytokines TNFa, IL-1 $\beta$ and IL6 in the colon mucosa of female mice. These cytokines are principally secreted by immune cells involved in innate immunity, whereas no differences were observed in the proinflammatory cytokine secretion of IFNy (Th1) and IL-17 (Th17) from adaptive immune cells. Moreover, no significant changes were noticed regarding the production of the anti-inflammatory cytokines IL-10 and TGF $\beta$ in the colon that normally increase in the case of mucosal inflammation $[79,80]$. Altogether, these data indicated that 13 weeks of exposure to a low dose of E175 relevant for humans (i.e., $1 \mu \mathrm{g} / \mathrm{kg} \mathrm{BW} / \mathrm{d}$ ) activated innate immune cells in the colon of female mice only, leading to low-grade (TNFa-, IL-1 $\beta$ - and IL-6-mediated) inflammation, as evidenced by the low levels of faecal Lcn-2. One may hypothesize that the increased production of TNFa and IL- 6 in the female colon could be linked to the decreased SCFA produced by the gut microbiota, which are known to inhibit histone deacetylases involved in the regulation of TNFa and IL- 6 secretion by macrophages [81].

In male mice, except for the proinflammatory cytokine IFNy, which was increased after exposure to the lowest E175 dose tested $(0.1 \mu \mathrm{g} / \mathrm{kg}$ BW/d), the colonic production of IL-1 $\beta$, IL- 6 and IL-17 and the antiinflammatory cytokine TGF $\beta$ were significantly decreased after the treatment period. These data clearly show that in males, chronic exposure to E175 at human dietary levels reduced intestinal immune activity, in contrast to females. As E175 exposure affected both the pro- and anti-inflammatory cytokine pathways, this did not result in intestinal inflammation under basal conditions, as indicated by the low levels of faecal Lcn-2. We suggest that the decreased production of IL-17 in the male colon exposed to E175 could be partly due to the impaired ability of the microbiota to catabolize tryptophan into AhR ligands. Indeed, indole derivates, which are tryptophan catabolites generated by the gut microbiota, were identified as activators of AhR and are highly expressed on Th17 and innate lymphoid cells group 3 that produce IL-17 [56]. Any modification in AhR ligand production by the microbiota impacts IL-17 levels and therefore acts on the fragile equilibrium between the microbiota and the host cells. Accordingly, we reported in the present study that the capacity of the gut microbiota to activate AhR was decreased following E175 exposure. Furthermore, this defect in AhR activation was observed in male mice only, which confirmed the sexual dimorphism noted in the intestinal production of IL-17 after long-term exposure to the food additive. It has been shown that IL-17 plays a protective role against muco-epithelial bacterial infection [82], which suggests that male mice exposed daily to E175 could be more susceptible to bacterial pathogens due to the drop in IL-17 in the gut mucosa. Interestingly, similar sex-related differences in inflammation profiles have been reported following exposure to polyethylene glycol-coated Au-NPs [83], where a significant increase in the spleen index, an indicator of immune response activation, was demonstrated in females but not in males. Under basal conditions, the innate and adaptive immune 
responses are stronger in females than in males, and studies have extended the notion of the sexual dimorphism to the gut microbiota [51-53]. Altogether, this could partly explain the differences observed between male and female mice following chronic exposure to Ref-Au or edible gold as food additive E175.

\section{Conclusions}

Our study reports that a 90-day E175 exposure in mice at human dietary levels from a solid matrix neither induces histomorphological damage in the liver, spleen and intestine nor genotoxic effects in the colon and liver. A higher systemic passage of Au particles was observed in mice exposed to a Ref-Au compared to E175, suggesting a size-dependent absorption of Au particles in the gut. However, due to the very low absorption level, which is in line with previous studies, we concluded that regardless of their size, most ingested Au particles accumulate in the intestinal lumen, where they can modulate the intricate dialogue between the gut microbiota and intestinal immune cells. After 13 weeks of exposure to E175, a more marked alteration of the gut microbiota composition and activity, characterized by an increased F/B ratio and Proteobacteria abundance as well as a decreased production of SCFAs, was noticed in female mice compared to males. Of note, similar gut microbiota alterations have been reported in mice and humans with metabolic disorders. On this basis, one may hypothesize that such alterations in E175-exposed mice, when associated with low-grade inflammation, as herein shown in the colon of females, could promote aggravation of metabolic disorders in individuals, for example, under an unbalanced diet. Due to a sexual dimorphism in the E175-related effects, the gut microbiota composition in males was less impacted than that in females by chronic exposure to the food additive. We suggest that the decreased capacity of the gut microbiota to produce SCFAs and AhR ligands could be partly responsible for the overall depression of intestinal immune status in male mice. Finally, in both sexes, E175 effects on the gut microbiota and/or intestinal immune response started at doses in the range of dietary exposure levels in humans and mostly appeared as nontoxic for the host. However, aggravation of gut dysbiosis in females at the higher dosage led us to suggest a potential risk of promoting metabolic disorders. Considering this potential hazard to human health and the use ad quatum satis of the E175 in the food industry, the establishment of toxic reference values for the safe use of gold as food additive (E175) should be considered in the human diet.

\section{Material And Methods}

\section{Gold reference nanoparticle and E175 characterization}

The E175 sample was obtained from a French commercial supplier of food colouring (OR A DECOR, La Chapelle Rambaud, France) as a representative batch of edible gold used as a food additive in the EU [5]. Its production was obtained from thin gold sheets that were then reduced by a milling process to gold flakes for commercial use as food additives. The reference Au test nanomaterial (Ref-Au) was purchased from SkySpring Nanomaterials Inc. (Houston, Texas, USA) and ranged in size from 50 to $100 \mathrm{~nm}$. The dimensional properties of the E175 sample were analysed by scanning electron microscopy (SEM). The 
E175 sample preparation protocol for SEM measurements was as follows: a quantity of powder (approximately $10 \mathrm{mg}$ ) was mixed with $5 \mathrm{~mL}$ of acetone, and the obtained suspension was homogenized with a vortex mixer. Then, a drop of the suspension was deposited on a silicon substrate by speedcoating. This deposition protocol comprises two phases: (1) spreading a drop of suspension over the silicon substrate with a low rotation speed and (2) rapid drying of the drop at a high rotation speed. The SEM equipment used was a Zeiss UltraPlus combining a high-tech Gemini FEG (Field Emission Gun) column with an in-lens detector located within the column (LNE, Trappes, France). The sample consists of gold foils with lateral dimensions ranging from several tens to hundreds of micrometres. In the present study, the accurate E175 dimension measured by SEM corresponds to the thickness of these sheets. Thickness measurements were performed on fragments of foils in several images. The method used was as follows: (1) different fragments of foils with apparent edges were selected and imaged; (2) measurements of thickness were performed on several points of the edge, with these points being regularly spaced; and (3) a number size distribution histograms were constructed from the thickness measurements (172 thickness measurements were performed on different fragments of foils to construct the histogram). The measurements were carried out with the PlatypusTM software platform from Pollen Metrology. Chemical elemental analysis of the E175 and Ref-Au powders was performed by transmission electronic microscopy (TEM) coupled to energy-dispersive X-ray (EDX) spectroscopy. The powders were diluted in water, and a few drops were deposited either on a copper membrane grid for TEM observation or on a nickel grid for EDX analysis. The sections were observed under a JEOL JEM-1400 electron microscope (MeTi facility, Toulouse, France) for TEM observations and were analysed by EDX under a JEOL $2100 \mathrm{~F}$ for chemical elemental analysis (Raimond Castaing platform, Toulouse, France).

\section{Animals and treatments}

Four-week-old male (M) and female (F) C57BL/6J mice were purchased from Janvier (France) and acclimated for 1 week before use. Throughout the study, the mice were maintained in polysulfone cages in a pathogen-free environment maintained at $22 \pm 2{ }^{\circ} \mathrm{C}$ under a 12-h light-dark cycle. Experimental design was based on a repeated dose (90-day) oral toxicity OECD study according to the guidance on risk assessment of nanomaterials in the food and feed chain [59]. Mice were randomly assigned to experimental groups ( $n=10 / \mathrm{sex} /$ condition) and fed ad libitum for 13 weeks with untreated diet $(0 \mu \mathrm{g} / \mathrm{kg}$ $\mathrm{BW} / \mathrm{d}$ ) or with the E175 food additive incorporated into the food pellets at relevant human dose levels of 0.1 and $1 \mu \mathrm{g} / \mathrm{kg} \mathrm{BW} / \mathrm{d}$ and at a high dose level of $10 \mu \mathrm{g} / \mathrm{kg} \mathrm{BW} / \mathrm{d}$. One supplemental group of mice $(\mathrm{n}=10$ animals/sex) was treated for 13 weeks with a Ref-Au nanomaterial (100\% nanosized, incorporated into the food pellets) at the higher dose level of $10 \mu \mathrm{g} / \mathrm{kg}$ BW/d for comparison of the potential size effect of $\mathrm{Au}$ particles. Mice were weighed every two or three days, and food consumption was recorded per cage to calculate the actual E175 or Ref-Au daily intake.

\section{Examination of particle absorption by confocal microscopy}

Tissue samples were embedded in paraffin wax and sectioned at a thickness of approximately $5 \mu \mathrm{m}$. Tissue sections were incubated with WGA-Alexa 594 for $1 \mathrm{~h}$ in the dark and then washed before being 
mounted in ProLong Gold antifade mounting medium (Life Technologies, France) containing DAPI (4',6diamidino-2-phenylindole) for fluorescence microscopy. Slides were viewed using a Leica SP8 confocal microscope for laser reflection particle detection with the 40x immersion objective as described previously [12]. Briefly, tissue sections were examined at 488/BP 488-494 $\mathrm{nm}$ to detect laser reflection by metal particles and at 514/BP 560-660 nm to visualize WGA staining in the tissue. Inorganic (mainly metal) particles appeared in green (laser reflection), cell nuclei appeared in blue (DAPI staining), and glycosylated parts of cell membranes and mucus cells appeared in red (WGA-Alexa 594 staining).

\section{Transmission electron microscopy and EDX analysis}

Tissue samples were embedded in Spurr resin as described previously [12]. The samples in resin blocks were cut with a Leica ultramicrotome, and 80-nm-thick sections were deposited on copper grids and then stained with a UAR-EMS (Uranyl Acetate Replacement) solution followed by a $0.4 \%$ lead citrate solution. The sections were observed under an electron microscope operated at $200 \mathrm{kV}$ using a JEOL JEM-1400 electron microscope (MeTi facility, Toulouse, France) for transmission electronic microscopy (TEM) observations and were analysed by energy-dispersive X-ray spectroscopy (EDX) under a JEOL 2100F for chemical elemental analysis (Raimond Castaing platform, Toulouse, France).

\section{Ussing Chamber Experiments}

Following mouse euthanasia, ileum and colon segments were used to assess barrier function as described previously [12]. Intestinal strips were mounted in Ussing chambers (Physiological Instruments) with an aperture size of $0.125 \mathrm{~cm}^{2}$ and bathed in $1 \mathrm{ml}$ of circulating oxygenated Krebs buffer at $37^{\circ} \mathrm{C}$. $\mathrm{Ag}-\mathrm{AgCl}$ electrodes were used as short-circuiting electrodes and to measure the transepithelial electrical properties of the tissues throughout the experiment, including the transepithelial potential difference, short-circuit current, and transepithelial resistance (TER), permitting the assessment of tissue viability. After 20 min of equilibration, the paracellular permeability was assessed by measuring the mucosal to serosal flux of FITC-labelled 4-kDa dextran (Sigma) for 1 hour, and the results are expressed as the flux of dextran crossing $1 \mathrm{~cm}^{2}$ of epithelium per hour $\left(\mathrm{nmol} \cdot \mathrm{cm}^{-2} \cdot \mathrm{h}^{-1}\right)$.

\section{Histology}

Collected samples were fixed in $4 \%$ formalin for 24 hours prior to paraffin embedding. The $5-\mu \mathrm{m}$ sections were stained with haematoxylin-eosin and examined using light microscopy (90i Nikon microscope). Scoring was blindly performed following the method for the general assessment of intestinal and colonic inflammation that has been previously described [84]. The scoring is based on the visual quantification of inflammatory cell infiltration and the determination of epithelial alteration levels and mucosal structures. For the liver and spleen, histomorphological changes were recorded as previously described [85].

\section{Cytokine quantification}


Colon segments were homogenized in RIPA buffer ( $1 \%$ Igepa, $0.5 \%$ deoxycholic acid and $0.1 \%$ SDS in Tris buffered saline solution 1x; $\mathrm{pH}$ 7.4) with protease inhibitors (Roche Diagnostics) and centrifuged at $10,000 \mathrm{~g}$ for $4 \mathrm{~min}\left(4^{\circ} \mathrm{C}\right)$. Protein concentrations were assessed using a Coomassie protein assay kit (Thermo Scientific) according to the manufacturer's instructions. All samples were diluted in PBS to obtain a solution of protein at $1 \mathrm{mg} / \mathrm{mL}$ and stored at $-80^{\circ} \mathrm{C}$ until processing. ELISAs were performed on the supernatants to quantify the following mouse cytokines according to the manufacturer's instructions: IL-10, IL-17A, TNFa and IFNy (Mabtech, Nacka Strand, Sweden); IL-6, IL-1 $\beta$ and TGF $\beta$ (R\&D Systems, Minneapolis, MN, USA).

\section{Quantification of faecal lipocalin-2 levels}

Frozen faecal samples were weighed and suspended in cold PBS to determine the faecal lipocalin-2 (Lcn2) level using a DuoSet murine LCN2 ELISA kit (R\&D Systems) as described previously [86].

\section{Gene expression analysis}

Total RNA was isolated from colon samples using an RNeasy Mini Kit (Qiagen) according to the manufacturer's instructions. Quantitative RT-PCR was performed using SuperScript II Reverse Transcriptase (Life Technologies, Saint Aubin, France) and then a Takyon SYBR Green PCR kit (Eurogentec, Liège, Belgium) with specific mouse oligonucleotides. The primers used were as follows: Gapdh (sense) 5'-AACTTTGGCATTGTGGAAGG-3', (antisense) 5'-ACACATTGGGGGTAGGAACA-3'; IL-1 $\beta$ (sense) 5'-GCCCATCCTCTGTGACTCAT-3', (antisense) 5'-AGGCCACAGGTATTTTGTCG-3'; IL-17 (sense) 5'TTTAACTCCCTTGGCGCA-3', (antisense) 5'-CTTTCCCTCCGCATTGACAC-3'; TNFa (sense) 5'TCCCCAAAGGGATGAGAAGTTC-3', (antisense) 5'-GCGCTGGCTCAGCCACT-3'; IFNy (sense) 5'ATGAACGCTACACACTGCATC-3', (antisense) 5'-CCATCCTTTTGCCAGTTCCTC-3'; IL-10 (sense) 5'AGAAGCATGGCCCAGAAATCA-3', (antisense) 5'-GGCCTTGTAGACACCTTGGT-3'; and TGF $\beta$ (sense) 5'ACTGGAGTTGTACGGCAGTG-3', (antisense) 5'-GGATCCACTTCCAACCCAGG-3'. We used the $2^{-\triangle \triangle C t}$ quantification method with mouse Gapdh as an endogenous control and the group fed with untreated diet as a calibrator.

\section{Gut microbiota composition analysis}

Faecal DNA was extracted from weighted stool samples according to [39]. Briefly, faecal samples were resuspended for $10 \mathrm{~min}$ in $250 \mu \mathrm{l}$ of $4 \mathrm{M}$ guanidine thiocyanate in $0.1 \mathrm{M}$ Tris (pH 7.5) (Sigma-Aldrich) and $40 \mu \mathrm{l}$ of $10 \% \mathrm{~N}$-lauroyl sarcosine (Sigma-Aldrich). After the addition of $500 \mu \mathrm{l}$ of $5 \% \mathrm{~N}$-lauroyl sarcosine in $0.1 \mathrm{M}$ phosphate buffer $(\mathrm{pH} 8.0)$, the samples were incubated at $70{ }^{\circ} \mathrm{C}$ for $1 \mathrm{~h}$. Sterilized silica beads were added, and the tube was shaken at $6.5 \mathrm{~m} / \mathrm{s}$ three times for $30 \mathrm{~s}$ each in a FastPrep (MP Biomedicals) apparatus. Then, polyvinylpolypyrrolidone $(15 \mathrm{mg})$ was added to the tube prior to centrifugation ( $5 \mathrm{~min}$ at $20,000 \mathrm{~g}$ ). After recovery of the supernatant, the pellets were washed with TENP (50 mM Tris (pH 8), $20 \mathrm{mM}$ EDTA ( $\mathrm{pH}$ 8), $100 \mathrm{mM} \mathrm{NaCl}$, and 1\% polyvinylpolypyrrolidone). The new supernatant was added to the first supernatant. The washing step was repeated two times. Nucleic acids were precipitated by the addition of isopropanol and centrifugation (10 min at 20,000 g). Pellets were 
resuspended in $450 \mu \mathrm{l}$ of $100 \mathrm{mM}$ phosphate buffer, $\mathrm{pH} 8$, and $50 \mu \mathrm{l}$ of $5 \mathrm{M}$ potassium acetate. The tube was placed on ice overnight and centrifuged at $20,000 \mathrm{~g}$ for $30 \mathrm{~min}$. The supernatant was then transferred to a new tube containing $20 \mu \mathrm{l}$ of RNase $(1 \mathrm{mg} / \mathrm{ml})$ and incubated at $37^{\circ} \mathrm{C}$ for $30 \mathrm{~min}$. Nucleic acids were precipitated by the addition of $50 \mu$ of $3 \mathrm{M}$ sodium acetate and $1 \mathrm{ml}$ of absolute ethanol. Nucleic acids were recovered by centrifugation at $20,000 \mathrm{~g}$ for $15 \mathrm{~min}$. The DNA pellet was finally washed three times with $70 \%$ ethanol, dried, and resuspended in $100 \mu \mathrm{l}$ of Tris-EDTA (TE) buffer. DNA extract quantity and quality were measured using a Nanodrop spectrophotometer (Thermo Scientific) prior to storage at $-80^{\circ} \mathrm{C}$.

The 16S rRNA genes, region V4, were PCR amplified using the $515 \mathrm{~F}$ (5'-GTGYCAGCMGCCGCGGTAA3')/806 R (5'-GGACTACNVGGGTWTCTAAT-3') primer pair and the following PCR procedure: 30 cycles of $45 \mathrm{~s}$ at $95^{\circ} \mathrm{C}, 60 \mathrm{~s}$ at $50^{\circ} \mathrm{C}$ and $90 \mathrm{~s}$ at $72{ }^{\circ} \mathrm{C}$ on a thermocycler. The sequencing of amplicons was performed using Illumina MiSeq technology (paired-end reads, 2 × $250 \mathrm{bp}$ ). The obtained data were processed using the quantitative insights into microbial ecology (QIIME 2) software package. All amplicon sequence variants (ASVs) were aligned using MAFFT and used to construct a phylogeny with FastTree 2 [87]. The taxonomy was assigned to each ASV using the 'classify-sklearn' command in q2featureclassifier against the Greengenes reference database (version 13.8). Alpha diversity metrics (richness and Shannon index) and beta diversity metrics (Bray-Curtis) were calculated using the q2diversity command. The differentially abundant bacterial taxa were determined at the genus or species level using analysis of composition of microbiomes (ANCOM) [88].

\section{Measurement of intestinal AhR activity}

The AhR activity of mouse stool samples was measured using a luciferase reporter assay method, as previously described [39]. Briefly, the H1L1.1c2 cell line, containing a stably integrated dioxin response element-driven firefly luciferase, was seeded in a 96-well plate and stimulated with mouse stool suspensions for $24 \mathrm{~h}$. Luciferase activity was measured using a luminometer, and the results are reported as fold changes based on the luciferase activity of an unstimulated control. All values were normalized on the basis of the cytotoxicity of the samples using the Lactate Dehydrogenase Activity Assay (Promega).

\section{Fecal metabolic profile using ${ }^{1} \mathrm{H}-\mathrm{NMR}$ spectroscopy}

Faecal aqueous extracts were prepared for NMR analysis as previously described $[89,90]$. All ${ }^{1} \mathrm{H}$ NMR spectra were obtained on a Bruker DRX-600-Avance NMR spectrometer (Bruker) using the MetaToulAXIOM metabolomics platform (MetaboHUB), operating at $600.13 \mathrm{MHz}$ for the ${ }^{1} \mathrm{H}$ resonance frequency, with an inverse-detection 5-mm H1-C13-N15 cryoprobe attached to a cryoplatform (the preamplifier cooling unit). The resultant spectra were phased, baseline corrected, and manually calibrated to trimethylsilylpropanoic acid (TSP) ( 80.00 ppm) using Mnova NMR (version 9.0; Mestrelab Research S.L.). After removing the region containing the water resonance $(\delta 4.6-5.2 \mathrm{ppm})$, the spectra were normalized to the probabilistic quotient [91] and aligned [92]. Data were mean-centred and scaled using unit variance 
scaling, followed by analysis with orthogonal projection on latent structure-discriminant analysis (O-PLSDA). To identify metabolites responsible for discrimination between the groups, we calculated the 0-PLSDA correlation coefficients ( $r 2$ ) for each variable and back-scaled them into a spectral domain, thus preserving the NMR spectra shapes and the coefficient signs $[93,94]$. We filtered the correlation coefficients extracted from significant models, such that correlations were only considered significant if they exceeded the threshold defined by Pearson's critical correlation coefficient $(p<0.05 ;|r|>0.7$; for $n=10$ per group). For illustration purposes, the area under the curve for significant metabolites was integrated.

\section{Genotoxic assessment}

Cells from intestinal and liver tissues were isolated for genotoxicity determination. Briefly, cells from the intestinal tissue segments (size between 1 and 1.5 centimetres) from the different test groups were isolated by longitudinally opening the piece of intestine with scissors and scraping the internal surface of intestine using a scalpel blade. The cells were then dissociated by incubation at $37^{\circ} \mathrm{C}$ for 30 minutes in a solution of collagenase and collected in PBS after 5 minutes of centrifugation and aspiration of the supernatant. For the liver segments (weight between 100 and $200 \mathrm{mg}$ ), cells were isolated by cutting the sample into small pieces with a scalpel prior to dissociation by incubation at $37^{\circ} \mathrm{C}$ for 30 minutes in a solution of collagenase. After several steps of manual dissociation and centrifugation, the isolated cells were collected in PBS. To ensure the same number of cells for all the samples, the cells isolated from the colons and livers of different groups were counted with a cell counter to produce a cell suspension at 200,000 cells/ $\mathrm{ml}$ before being deposited in duplicate in 96-well plates (40,000 cells/well).

The $\mathrm{YH} 2 \mathrm{AX}$ genotoxicity assay was performed with the in-cell western (ICW) technique as previously described [95-98]. Briefly, the cells in 96-well plates were fixed with paraformaldehyde (Electron Microscopy Science), permeabilized with $0.1 \%$ Triton X-100 (Sigma-Aldrich) in PBS and blocked with MAXblock Blocking Medium (Active Motif) before being washed with PBS supplemented with $2 \%$ foetal calf serum and $0.1 \%$ Triton X-100 (PST buffer). Cells were then incubated with rabbit monoclonal antiYH2AX primary antibody (Clone 20E3, Cell Signaling) in PST buffer. After three washes in PST buffer, secondary detection was carried out using an infrared fluorescent dye conjugated to goat antibody (CF770, Biotium). For DNA labelling, RedDot2 (Biotium) labelling was used. After incubation followed by three washes, DNA and the biomarker of interest $(\mathrm{\gamma H} 2 \mathrm{AX})$ were simultaneously visualized using an Odyssey Infrared Imaging Scanner (LiCor ScienceTec, Les Ulis, France). For the determination of genotoxicity, relative fluorescent units for $\mathrm{YH} 2 \mathrm{AX}$ per cell (as determined by the $\mathrm{YH} 2 \mathrm{AX}$ content divided by the DNA content) were divided by the respective controls (vehicle only) to determine the change in the level of phosphorylation of histone H2AX compared with the control group.

\section{Statistical analysis}

Data visualization and statistical analysis were conducted using GraphPad Prism version 9.0 (GraphPad software, Sand Diego, USA). Statistical analysis for the intestinal permeability measurements, intestinal immune response and gut microbiota analysis were conducted as follows: 
Normal distribution was verified using the Kolmogorov-Smirnov test with Dallal-Wilkinson-Lillie correction. Statistical differences were determined using one-way analysis of variance (ANOVA) followed by the post hoc Tukey's test for multiple comparisons. When assumptions of a normal distribution were not met, a nonparametric Kruskal-Wallis test was conducted, followed by a post hoc Dunn's test. For comparisons between two groups, significant differences were determined using the two-tailed Student's $\mathrm{t}$ test or nonparametric Mann-Whitney test. In all determinations, statistical outliers (using the 18 ROUT method) or samples where technical issues were encountered, such as poor RNA quality, poor tissue quality for permeability measurements, or poor histological orientation, were removed from analysis. Specifically, for the analysis of the gut microbiota beta-diversity, PERMANOVA was applied using the distances dataset to compare the centroids and dispersion of the experimental groups. If significant differences were indicated ( $p$ value<0.05), pairwise comparisons were performed to determine the conditions that were significantly different from the control group.

For metabolite production analysis, the area under the curve of metabolites was tested for statistical significance using one-way analysis of variance (ANOVA) followed by Sidak's post-test for multiple comparisons. Metabolites with both a significant correlation coefficient from the predictive axis on the 0PLS-DA models and a significant $p$ value using univariate statistics were considered significantly different between groups.

\section{Abbreviations}

AhR: aryl hydrocarbon receptor; ANOVA: one-way analysis of variance; BW: body weight; EDX: energydispersive X-ray; EFSA: European Food Safety Authority; EU: European union; F/B :

Firmicutes/Bacteroidetes; FITC: fluorescein isothiocyanate; Gapdh: Glyceraldehyde 3-phosphate dehydrogenase; IFN: Interferon; IL: Interleukin; Lcn: Lipocalin; MCFA: Medium-chain fatty acids; NMR: Nuclear magnetic resonance; NP: nanoparticle; OPLS-DA: Orthogonal partial least squares discriminant analysis; Ref: reference; RT-PCR: Reverse transcriptase Polymerase chain reaction ; SCFA: short-chain fatty acids; TEM: Transmission electron microscopy; TER: Transepithelial resistance; TGF: transforming growth factor; TNF: tumor necrosis factors; WGA: Wheat Germ Agglutinin

\section{Declarations}

Ethics approval and consent to participate: All animal experiments were performed in accordance with the guidelines of European legislation (Council Directive 2010/63/UE) and French Decree 2013-118 on the protection of animals used for scientific purposes and were approved by the Local Animal Care and Use Committee (TOXCOM-0236) of Occitanie Toulouse (agreement CEEA-86). The animal facilities at INRAe Toxalim are licenced by the relevant local authorities for rodents (agreement C31 555 13).

Consent for publication: All authors read and approved the final manuscript. 
Availability of data and materials: All relevant data are included in the manuscript and supporting information, and available from the authors upon request.

Competing interests: MA and LK are co-founders of Preditox SAS, a company specialised in genotoxicity prediction. LK is CEO and MA serves as consultant to Preditox SAS. The authors declare that they have no competing interests.

Funding: The study was supported by INRAe (National research institute for agriculture, food and environment) under the research contract with OR A DECOR SARL acting in its own name and/or on behalf consortium of producers of E175 food additives and users, in the frame of the EFSA's 2018 call for scientific and technical data on the permitted food additive gold (E175).

Author's contributions: $\mathrm{BL}, \mathrm{MA}$ and $\mathrm{EH}$ conceived and designed the study; $\mathrm{LE}, \mathrm{BL}$ and $\mathrm{EH}$ performed data analysis and wrote the manuscript; $B L, E G$ and $C C$ conducted all experiments, unless otherwise indicated; $\mathrm{BL}, \mathrm{LE}$ and SES performed ${ }^{1} \mathrm{H}-\mathrm{NMR}$ analysis; NF, LD and GF performed SEM analysis; LK and MA performed Genotoxic assessment; $L E, B L$ and $B C$ performed microbiota composition analysis; $L E, B L, M A$ and $\mathrm{EH}$ discussed the experiments and results.

Acknowledgements: The authors thank the employees of the animal facility at INRAe and G Chevalier for scientific advices as well as Natalia Martins Breyner, Adèle Guillard and Aurélie Pettes-Duler at UMR 1331 Toxalim (Toulouse, France) and Charlène Dauriat at INSERM U1016 for their technical help.

\section{References}

1. Waegeneers N, De Vos S, Verleysen E, Ruttens A, Mast J. Estimation of the Uncertainties Related to the Measurement of the Size and Quantities of Individual Silver Nanoparticles in Confectionery. Materials (Basel). 2019;12.

2. De Vos S, Waegeneers N, Verleysen E, Smeets K, Mast J. Physico-chemical characterisation of the fraction of silver (nano)particles in pristine food additive E174 and in E174-containing confectionery. Food Addit Contam Part A Chem Anal Control Expo Risk Assess. 2020;37:1831-46.

3. Verleysen E, Waegeneers N, Brassinne F, De Vos S, Jimenez IO, Mathioudaki S, et al. Physicochemical Characterization of the Pristine E171 Food Additive by Standardized and Validated Methods. Nanomaterials. Multidisciplinary Digital Publishing Institute; 2020;10:592.

4. Wittsiepe J, Schrey P, Wilhelm M, Begerow J, Dunemann L. Dietary intake of platinum and gold by children from Germany using duplicate portion sampling. Journal of Trace Elements in Medicine and Biology. 2003;17:117-22.

5. EFSA. Scientific Opinion on the re-evaluation of gold (E 175) as a food additive. EFSA Journal. 2016;14:4362.

6. Downham A, Collins P. Colouring our foods in the last and next millennium. International Journal of Food Science \& Technology. 2000;35:5-22. 
7. Lamas B, Martins Breyner N, Houdeau E. Impacts of foodborne inorganic nanoparticles on the gut microbiota-immune axis: potential consequences for host health. Particle and Fibre Toxicology. 2020;17:19.

8. Medina-Reyes El, Rodríguez-Ibarra C, Déciga-Alcaraz A, Díaz-Urbina D, Chirino YI, Pedraza-Chaverri J. Food additives containing nanoparticles induce gastrotoxicity, hepatotoxicity and alterations in animal behavior: The unknown role of oxidative stress. Food and Chemical Toxicology. 2020;146:111814.

9. Javurek AB, Suresh D, Spollen WG, Hart ML, Hansen SA, Ellersieck MR, et al. Gut Dysbiosis and Neurobehavioral Alterations in Rats Exposed to Silver Nanoparticles. Sci Rep. 2017;7:2822.

10. Radziwill-Bienkowska JM, Talbot P, Kamphuis JBJ, Robert V, Cartier C, Fourquaux I, et al. Toxicity of Food-Grade TiO2 to Commensal Intestinal and Transient Food-Borne Bacteria: New Insights Using Nano-SIMS and Synchrotron UV Fluorescence Imaging. Frontiers in Microbiology. 2018;9:794.

11. Yamashita K, Yoshioka Y, Higashisaka K, Mimura K, Morishita Y, Nozaki M, et al. Silica and titanium dioxide nanoparticles cause pregnancy complications in mice. Nature Nanotechnology. Nature Publishing Group; 2011;6:321-8.

12. Coméra C, Cartier C, Gaultier E, Catrice O, Panouille Q, El Hamdi S, et al. Jejunal villus absorption and paracellular tight junction permeability are major routes for early intestinal uptake of food-grade TiO2 particles: an in vivo and ex vivo study in mice. Particle and Fibre Toxicology. 2020;17:26.

13. Guillard A, Gaultier E, Cartier C, Devoille L, Noireaux J, Chevalier L, et al. Basal Ti level in the human placenta and meconium and evidence of a materno-foetal transfer of food-grade TiO2 nanoparticles in an ex vivo placental perfusion model. Part Fibre Toxicol. 2020;17:51.

14. Pan Y, Neuss S, Leifert A, Fischler M, Wen F, Simon U, et al. Size-dependent cytotoxicity of gold nanoparticles. Small. 2007;3:1941-9.

15. Xia Q, Li H, Liu Y, Zhang S, Feng Q, Xiao K. The effect of particle size on the genotoxicity of gold nanoparticles. Journal of Biomedical Materials Research Part A. 2017;105:710-9.

16. Vales G, Suhonen S, Siivola KM, Savolainen KM, Catalán J, Norppa H. Genotoxicity and Cytotoxicity of Gold Nanoparticles In Vitro: Role of Surface Functionalization and Particle Size. Nanomaterials. Multidisciplinary Digital Publishing Institute; 2020;10:271.

17. Chithrani BD, Ghazani AA, Chan WCW. Determining the Size and Shape Dependence of Gold Nanoparticle Uptake into Mammalian Cells. Nano Lett. American Chemical Society; 2006;6:662-8.

18. Ma X, Wu Y, Jin S, Tian Y, Zhang X, Zhao Y, et al. Gold nanoparticles induce autophagosome accumulation through size-dependent nanoparticle uptake and lysosome impairment. ACS Nano. 2011;5:8629-39.

19. Hornos Carneiro MF, Barbosa F. Gold nanoparticles: A critical review of therapeutic applications and toxicological aspects. J Toxicol Environ Health B Crit Rev. 2016;19:129-48.

20. Balogh L, Nigavekar SS, Nair BM, Lesniak W, Zhang C, Sung LY, et al. Significant effect of size on the in vivo biodistribution of gold composite nanodevices in mouse tumor models. Nanomedicine. 2007;3:281-96. 
21. De Jong WH, Hagens WI, Krystek P, Burger MC, Sips AJAM, Geertsma RE. Particle size-dependent organ distribution of gold nanoparticles after intravenous administration. Biomaterials. 2008;29:1912-9.

22. Khlebtsov N, Dykman L. Biodistribution and toxicity of engineered gold nanoparticles: a review of in vitro and in vivo studies. Chem Soc Rev. The Royal Society of Chemistry; 2011;40:1647-71.

23. Zhang X-D, Wu D, Shen X, Liu P-X, Yang N, Zhao B, et al. Size-dependent in vivo toxicity of PEGcoated gold nanoparticles. Int J Nanomedicine. 2011;6:2071-81.

24. Lopez-Chaves C, Soto-Alvaredo J, Montes-Bayon M, Bettmer J, Llopis J, Sanchez-Gonzalez C. Gold nanoparticles: Distribution, bioaccumulation and toxicity. In vitro and in vivo studies. Nanomedicine: Nanotechnology, Biology and Medicine. 2018;14:1-12.

25. Adewale OB, Davids H, Cairncross L, Roux S. Toxicological Behavior of Gold Nanoparticles on Various Models: Influence of Physicochemical Properties and Other Factors. Int J Toxicol. SAGE Publications Inc; 2019;38:357-84.

26. Lee JK, Kim TS, Bae JY, Jung AY, Lee SM, Seok JH, et al. Organ-specific distribution of gold nanoparticles by their surface functionalization. J Appl Toxicol. 2015;35:573-80.

27. Schmid G, Kreyling WG, Simon U. Toxic effects and biodistribution of ultrasmall gold nanoparticles. Arch Toxicol. 2017;91:3011-37.

28. Zhang X-D, Wu H-Y, Wu D, Wang Y-Y, Chang J-H, Zhai Z-B, et al. Toxicologic effects of gold nanoparticles in vivo by different administration routes. Int J Nanomedicine. 2010;5:771-81.

29. Lasagna-Reeves C, Gonzalez-Romero D, Barria MA, Olmedo I, Clos A, Sadagopa Ramanujam VM, et al. Bioaccumulation and toxicity of gold nanoparticles after repeated administration in mice. Biochemical and Biophysical Research Communications. 2010;393:649-55.

30. Bahamonde J, Brenseke B, Chan MY, Kent RD, Vikesland PJ, Prater MR. Gold Nanoparticle Toxicity in Mice and Rats: Species Differences. Toxicol Pathol. SAGE Publications Inc; 2018;46:431-43.

31. Chen Y-S, Hung Y-C, Liau I, Huang GS. Assessment of the In Vivo Toxicity of Gold Nanoparticles. Nanoscale Res Lett. 2009;4:858-64.

32. Li J, Cha R, Zhao X, Guo H, Luo H, Wang M, et al. Gold Nanoparticles Cure Bacterial Infection with Benefit to Intestinal Microflora. ACS Nano. American Chemical Society; 2019;13:5002-14.

33. Jo M-R, Bae S-H, Go M-R, Kim H-J, Hwang Y-G, Choi S-J. Toxicity and Biokinetics of Colloidal Gold Nanoparticles. Nanomaterials (Basel). 2015;5:835-50.

34. Enea M, Pereira E, Silva DD, Costa J, Soares ME, de Lourdes Bastos M, et al. Study of the intestinal uptake and permeability of gold nanoparticles using both in vitro and in vivo approaches. Nanotechnology. 2020;31:195102.

35. Cho W-S, Cho M, Jeong J, Choi M, Cho H-Y, Han BS, et al. Acute toxicity and pharmacokinetics of 13 nm-sized PEG-coated gold nanoparticles. Toxicology and Applied Pharmacology. 2009;236:1624. 
36. Shrivastava R, Kushwaha P, Bhutia YC, Flora S. Oxidative stress following exposure to silver and gold nanoparticles in mice. Toxicol Ind Health. SAGE Publications Ltd STM; 2016;32:1391-404.

37. Ibrahim KE, Bakhiet AO, Awadalla ME, Khan HA. A priming dose protects against gold nanoparticlesinduced proinflammatory cytokines mRNA expression in mice [Internet]. https://doi.org/10.2217/nnm-2017-0332. Future Medicine Ltd London, UK; 2017 [cited 2021 May 19]. Available from: https://www.futuremedicine.com/doi/abs/10.2217/nnm-2017-0332

38. Kamada N, Seo S-U, Chen GY, Núñez G. Role of the gut microbiota in immunity and inflammatory disease. Nature Reviews Immunology. 2013;13:321-35.

39. Lamas B, Richard ML, Leducq V, Pham H-P, Michel M-L, Da Costa G, et al. CARD9 impacts colitis by altering gut microbiota metabolism of tryptophan into aryl hydrocarbon receptor ligands. Nature Medicine. Nature Publishing Group; 2016;22:598-605.

40. Rooks MG, Garrett WS. Gut microbiota, metabolites and host immunity. Nature Reviews Immunology. Nature Publishing Group; 2016;16:341-52.

41. Tomas J, Wrzosek L, Bouznad N, Bouet S, Mayeur C, Noordine M-L, et al. Primocolonization is associated with colonic epithelial maturation during conventionalization. FASEB J. 2013;27:645-55.

42. Natividad JM, Agus A, Planchais J, Lamas B, Jarry AC, Martin R, et al. Impaired Aryl Hydrocarbon Receptor Ligand Production by the Gut Microbiota Is a Key Factor in Metabolic Syndrome. Cell Metab. 2018;28:737-749.e4.

43. Dinan TG, Cryan JF. Gut instincts: microbiota as a key regulator of brain development, ageing and neurodegeneration. J Physiol. 2017;595:489-503.

44. Vijay-Kumar M, Aitken JD, Carvalho FA, Cullender TC, Mwangi S, Srinivasan S, et al. Metabolic syndrome and altered gut microbiota in mice lacking Toll-like receptor 5. Science. 2010;328:228-31.

45. Erny D, Hrabě de Angelis AL, Jaitin D, Wieghofer P, Staszewski O, David E, et al. Host microbiota constantly control maturation and function of microglia in the CNS. Nat Neurosci. 2015;18:965-77.

46. Rothhammer V, Mascanfroni ID, Bunse L, Takenaka MC, Kenison JE, Mayo L, et al. Type I interferons and microbial metabolites of tryptophan modulate astrocyte activity and central nervous system inflammation via the aryl hydrocarbon receptor. Nat Med. 2016;22:586-97.

47. Beaumont M, Neyrinck AM, Olivares M, Rodriguez J, de Rocca Serra A, Roumain M, et al. The gut microbiota metabolite indole alleviates liver inflammation in mice. The FASEB Journal. 2018;32:6681-93.

48. Endo H, Niioka M, Kobayashi N, Tanaka M, Watanabe T. Butyrate-Producing Probiotics Reduce Nonalcoholic Fatty Liver Disease Progression in Rats: New Insight into the Probiotics for the GutLiver Axis. PLOS ONE. Public Library of Science; 2013;8:e63388.

49. Sommer F, Bäckhed F. The gut microbiota - masters of host development and physiology. Nat Rev Microbiol. 2013;11:227-38.

50. Zhang Y, Shareena Dasari TP, Deng H, Yu H. Antimicrobial Activity of Gold Nanoparticles and lonic Gold. J Environ Sci Health C Environ Carcinog Ecotoxicol Rev. 2015;33:286-327. 
51. Org E, Mehrabian M, Parks BW, Shipkova P, Liu X, Drake TA, et al. Sex differences and hormonal effects on gut microbiota composition in mice. Gut Microbes. Taylor \& Francis; 2016;7:313-22.

52. Elderman M, Hugenholtz F, Belzer C, Boekschoten M, van Beek A, de Haan B, et al. Sex and strain dependent differences in mucosal immunology and microbiota composition in mice. Biology of Sex Differences. 2018;9:26.

53. Valeri F, Endres K. How biological sex of the host shapes its gut microbiota. Frontiers in Neuroendocrinology. 2021;61:100912.

54. Ratajczak W, Rył A, Mizerski A, Walczakiewicz K, Sipak O, Laszczyńska M. Immunomodulatory potential of gut microbiome-derived short-chain fatty acids (SCFAs). Acta Biochim Pol. 2019;66:112.

55. Chassaing B, Srinivasan G, Delgado MA, Young AN, Gewirtz AT, Vijay-Kumar M. Fecal Lipocalin 2, a Sensitive and Broadly Dynamic Non-Invasive Biomarker for Intestinal Inflammation. PLOS ONE. Public Library of Science; 2012;7:e44328.

56. Lamas B, Natividad JM, Sokol H. Aryl hydrocarbon receptor and intestinal immunity. Mucosal Immunology. Nature Publishing Group; 2018;11:1024-38.

57. Schiering C, Wincent E, Metidji A, Iseppon A, Li Y, Potocnik AJ, et al. Feedback control of AHR signalling regulates intestinal immunity. Nature. 2017;542:242-5.

58. Hadrup N, Sharma AK, Poulsen M, Nielsen E. Toxicological risk assessment of elemental gold following oral exposure to sheets and nanoparticles - A review. Regul Toxicol Pharmacol. 2015;72:216-21.

59. EFSA Scientific Committee, More S, Bampidis V, Benford D, Bragard C, Halldorsson T, et al. Guidance on risk assessment of nanomaterials to be applied in the food and feed chain: human and animal health. EFSA Journal. 2021;19:e06768.

60. Venkatpurwar V, Mali V, Bodhankar S, Pokharkar V. In vitro cytotoxicity and in vivo sub-acute oral toxicity assessment of porphyran reduced gold nanoparticles. Toxicological \& Environmental Chemistry. Taylor \& Francis; 2012;94:1357-67.

61. Rathore M, Mohanty IR, Maheswari U, Dayal N, Suman R, Joshi DS. Comparative in vivo assessment of the subacute toxicity of gold and silver nanoparticles. J Nanopart Res. 2014;16:2338.

62. Sonavane G, Tomoda K, Sano A, Ohshima H, Terada H, Makino K. In vitro permeation of gold nanoparticles through rat skin and rat intestine: Effect of particle size. Colloids and Surfaces B: Biointerfaces. 2008;65:1-10.

63. Fransen F, van Beek AA, Borghuis T, Meijer B, Hugenholtz F, van der Gaast-de Jongh C, et al. The Impact of Gut Microbiota on Gender-Specific Differences in Immunity. Frontiers in Immunology. 2017;8:754.

64. Schneeberger M, Everard A, Gómez-Valadés AG, Matamoros S, Ramírez S, Delzenne NM, et al. Akkermansia muciniphila inversely correlates with the onset of inflammation, altered adipose tissue metabolism and metabolic disorders during obesity in mice. Sci Rep. 2015;5:16643. 
65. Everard A, Belzer C, Geurts L, Ouwerkerk JP, Druart C, Bindels LB, et al. Cross-talk between Akkermansia muciniphila and intestinal epithelium controls diet-induced obesity. PNAS. National Academy of Sciences; 2013;110:9066-71.

66. Zhang T, Li Q, Cheng L, Buch H, Zhang F. Akkermansia muciniphila is a promising probiotic. Microb Biotechnol. 2019;12:1109-25.

67. LeBlanc JG, Chain F, Martín R, Bermúdez-Humarán LG, Courau S, Langella P. Beneficial effects on host energy metabolism of short-chain fatty acids and vitamins produced by commensal and probiotic bacteria. Microbial Cell Factories. 2017;16:79.

68. Mathur R, Barlow GM. Obesity and the microbiome. Expert Rev Gastroenterol Hepatol. 2015;9:108799.

69. Musso G, Gambino R, Cassader M. Obesity, diabetes, and gut microbiota: the hygiene hypothesis expanded? Diabetes Care. 2010;33:2277-84.

70. Ley RE, Turnbaugh PJ, Klein S, Gordon Jl. Human gut microbes associated with obesity. Nature. 2006;444:1022.

71. Lu Y, Chen J, Zheng J, Hu G, Wang J, Huang C, et al. Mucosal adherent bacterial dysbiosis in patients with colorectal adenomas. Sci Rep. 2016;6:26337.

72. Sokol H, Seksik P, Furet JP, Firmesse O, Nion-Larmurier I, Beaugerie L, et al. Low counts of Faecalibacterium prausnitzii in colitis microbiota. Inflamm Bowel Dis. 2009;15:1183-9.

73. Mariat D, Firmesse O, Levenez F, Guimarăes V, Sokol H, Doré J, et al. The Firmicutes/Bacteroidetes ratio of the human microbiota changes with age. BMC Microbiology. 2009;9:123.

74. Voreades N, Kozil A, Weir TL. Diet and the development of the human intestinal microbiome. Frontiers in Microbiology. 2014;5:494.

75. Shin N-R, Whon TW, Bae J-W. Proteobacteria: microbial signature of dysbiosis in gut microbiota. Trends in biotechnology. 2015;33:496-503.

76. Rizzatti G, Lopetuso LR, Gibiino G, Binda C, Gasbarrini A. Proteobacteria: A Common Factor in Human Diseases. BioMed Research International. 2017;2017:1-7.

77. Jia M, Zhang Y, Gao Y, Ma X. Effects of Medium Chain Fatty Acids on Intestinal Health of Monogastric Animals. Curr Protein Pept Sci. 2020;21:777-84.

78. Levy M, Thaiss CA, Elinav E. Metabolites: messengers between the microbiota and the immune system. Genes Dev. 2016;30:1589-97.

79. Ihara S, Hirata Y, Koike K. TGF- $\beta$ in inflammatory bowel disease: a key regulator of immune cells, epithelium, and the intestinal microbiota. J Gastroenterol. 2017;52:777-87.

80. Neurath MF. Cytokines in inflammatory bowel disease. Nat Rev Immunol. 2014;14:329-42.

81. Usami M, Kishimoto K, Ohata A, Miyoshi M, Aoyama M, Fueda Y, et al. Butyrate and trichostatin A attenuate nuclear factor kappaB activation and tumor necrosis factor alpha secretion and increase prostaglandin E2 secretion in human peripheral blood mononuclear cells. Nutr Res. 2008;28:321-8. 
82. Ishigame H, Kakuta S, Nagai T, Kadoki M, Nambu A, Komiyama Y, et al. Differential roles of interleukin-17A and -17F in host defense against mucoepithelial bacterial infection and allergic responses. Immunity. 2009;30:108-19.

83. Chen J, Wang H, Long W, Shen X, Wu D, Song S-S, et al. Sex differences in the toxicity of polyethylene glycol-coated gold nanoparticles in mice. Int J Nanomedicine. 2013;8:2409-19.

84. Erben U, Loddenkemper C, Doerfel K, Spieckermann S, Haller D, Heimesaat MM, et al. A guide to histomorphological evaluation of intestinal inflammation in mouse models. Int $\mathrm{J}$ Clin Exp Pathol. 2014;7:4557-76.

85. Bailly A-L, Correard F, Popov A, Tselikov G, Chaspoul F, Appay R, et al. In vivo evaluation of safety, biodistribution and pharmacokinetics of laser-synthesized gold nanoparticles. Scientific Reports. Nature Publishing Group; 2019;9:12890.

86. Lamas B, Michel M-L, Waldschmitt N, Pham H-P, Zacharioudaki V, Dupraz L, et al. Card9 mediates susceptibility to intestinal pathogens through microbiota modulation and control of bacterial virulence. Gut. 2018;67:1836-44.

87. Price MN, Dehal PS, Arkin AP. FastTree 2 - Approximately Maximum-Likelihood Trees for Large Alignments. PLOS ONE. Public Library of Science; 2010;5:e9490.

88. Mandal S, Van Treuren W, White RA, Eggesbø M, Knight R, Peddada SD. Analysis of composition of microbiomes: a novel method for studying microbial composition. Microb Ecol Health Dis. 2015;26:27663.

89. Beckonert O, Keun HC, Ebbels TMD, Bundy J, Holmes E, Lindon JC, et al. Metabolic profiling, metabolomic and metabonomic procedures for NMR spectroscopy of urine, plasma, serum and tissue extracts. Nature Protocols. Nature Publishing Group; 2007;2:2692-703.

90. Lukowicz C, Ellero-Simatos S, Régnier M, Polizzi A, Lasserre F, Montagner A, et al. Metabolic Effects of a Chronic Dietary Exposure to a Low-Dose Pesticide Cocktail in Mice: Sexual Dimorphism and Role of the Constitutive Androstane Receptor. Environ Health Perspect. 2018;126:067007.

91. Dieterle F, Ross A, Schlotterbeck G, Senn H. Probabilistic Quotient Normalization as Robust Method to Account for Dilution of Complex Biological Mixtures. Application in 1H NMR Metabonomics. Anal Chem. American Chemical Society; 2006;78:4281-90.

92. Veselkov KA, Lindon JC, Ebbels TMD, Crockford D, Volynkin VV, Holmes E, et al. Recursive SegmentWise Peak Alignment of Biological 1H NMR Spectra for Improved Metabolic Biomarker Recovery. Anal Chem. American Chemical Society; 2009;81:56-66.

93. Cloarec O, Dumas M-E, Craig A, Barton RH, Trygg J, Hudson J, et al. Statistical total correlation spectroscopy: an exploratory approach for latent biomarker identification from metabolic $1 \mathrm{H}$ NMR data sets. Anal Chem. 2005;77:1282-9.

94. Cloarec O, Dumas ME, Trygg J, Craig A, Barton RH, Lindon JC, et al. Evaluation of the orthogonal projection on latent structure model limitations caused by chemical shift variability and improved visualization of biomarker changes in $1 \mathrm{H}$ NMR spectroscopic metabonomic studies. Anal Chem. 2005;77:517-26. 
95. Graillot V, Tomasetig F, Cravedi J-P, Audebert M. Evidence of the in vitro genotoxicity of methylpyrazole pesticides in human cells. Mutat Res. 2012;748:8-16.

96. Graillot V, Takakura N, Hegarat LL, Fessard V, Audebert M, Cravedi J-P. Genotoxicity of pesticide mixtures present in the diet of the French population. Environ Mol Mutagen. 2012;53:173-84.

97. Khoury L, Zalko D, Audebert M. Validation of high-throughput genotoxicity assay screening using $\mathrm{YH} 2 \mathrm{AX}$ in-cell western assay on HepG2 cells. Environmental and Molecular Mutagenesis. 2013;54:737-46.

98. Khoury L, Zalko D, Audebert M. Evaluation of four human cell lines with distinct biotransformation properties for genotoxic screening. Mutagenesis. 2016;31:83-96.

\section{Figures}

\section{Figure 1}

Microscopy analysis of food-grade Au (E175) and Ref-Au particles. (A) Scanning electron microscopy (SEM) image of Au foils in an E175 water suspension. Scale bar $=100 \mu \mathrm{m}$. (B) EDX spectra of E175 particulate matter showing Au signals from particles and Ni from the nickel grid. (C) Number distribution histogram of the measured thicknesses of the fragments of Au foils from E175 powder. (D) Transmission electron microscopy (TEM) image of Au particles in a Ref-Au water suspension. Scale bar $=500 \mathrm{~nm}$. (E) EDX spectra of Ref-Au particulate matter showing Au signals from particles and Ni from the nickel grid.

\section{Figure 2}

Feed intake and body weight of mice exposed to Ref-Au or E175 for 13 weeks. (A) Daily feed intake of male mice exposed for 13 weeks to Ref-Au at $10 \mu \mathrm{g} / \mathrm{kg} \mathrm{BW} / \mathrm{d}$ or E175 at 0.1, 1 and $10 \mu \mathrm{g} / \mathrm{kg} \mathrm{BW} / \mathrm{d}$ incorporated in food pellets. (B) Body weight of male mice orally exposed for 13 weeks to Ref-Au at 10 $\mu \mathrm{g} / \mathrm{kg} \mathrm{BW} / \mathrm{d}$ or E175 at $0.1,1$ and $10 \mu \mathrm{g} / \mathrm{kg} \mathrm{BW} / \mathrm{d}$. (C) Daily feed intake of female mice exposed for 13 weeks to Ref-Au at $10 \mu \mathrm{g} / \mathrm{kg} \mathrm{BW} / \mathrm{d}$ or E175 at $0.1,1$ and $10 \mu \mathrm{g} / \mathrm{kg} \mathrm{BW} / \mathrm{d}$ incorporated in food pellets. (D) Body weight of female mice orally exposed for 13 weeks to Ref-Au at $10 \mu \mathrm{g} / \mathrm{kg}$ BW/d or E175 at 0.1, 1 and $10 \mu \mathrm{g} / \mathrm{kg} \mathrm{BW} / \mathrm{d}$. For statistical comparisons, the dagger ( $\dagger$ ) indicates Ref-Au versus control, asterisk (*) indicates E175 $0.1 \mu \mathrm{g} / \mathrm{kg}$ BW/d versus control, and double dagger $(\ddagger)$ indicates E175 $1 \mu \mathrm{g} / \mathrm{kg} B W / \mathrm{d}$ versus control. The data are expressed as the mean $\pm S E M$. $+P<0.05 ;{ }^{*} P<0.05 ;{ }^{* \star *} P<0.001 ; \neq \neq \neq P<0.001$; two-way ANOVA and post hoc Bonferroni test. 
Ref-Au
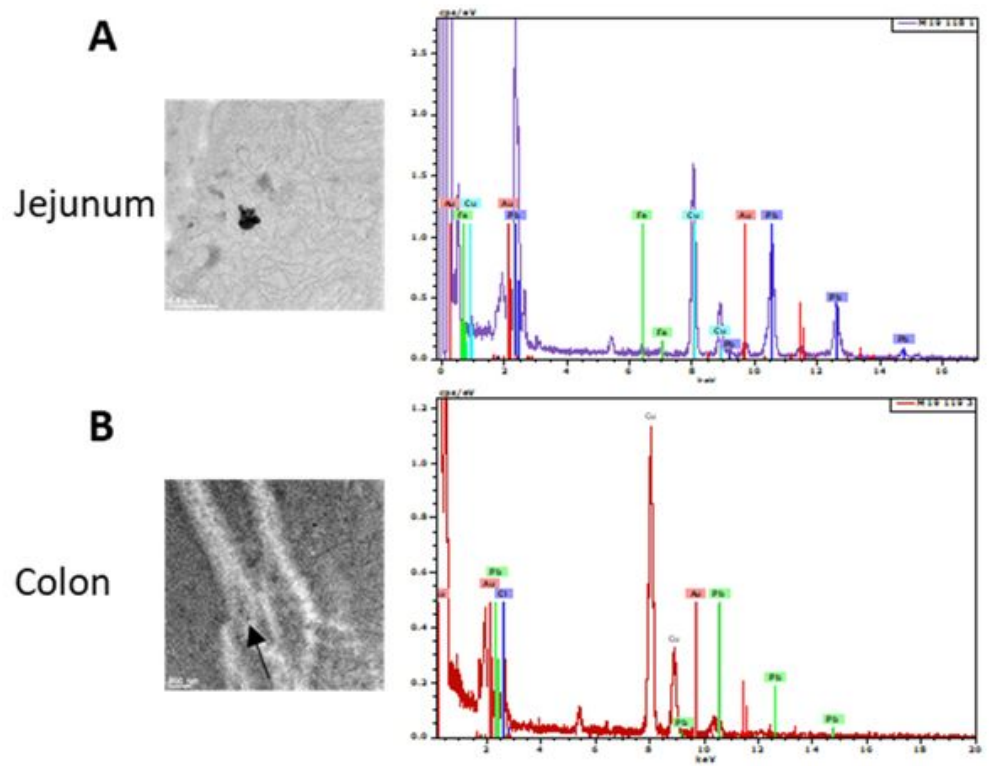

C

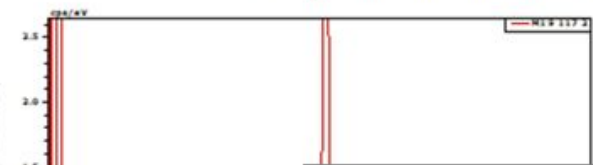

E175
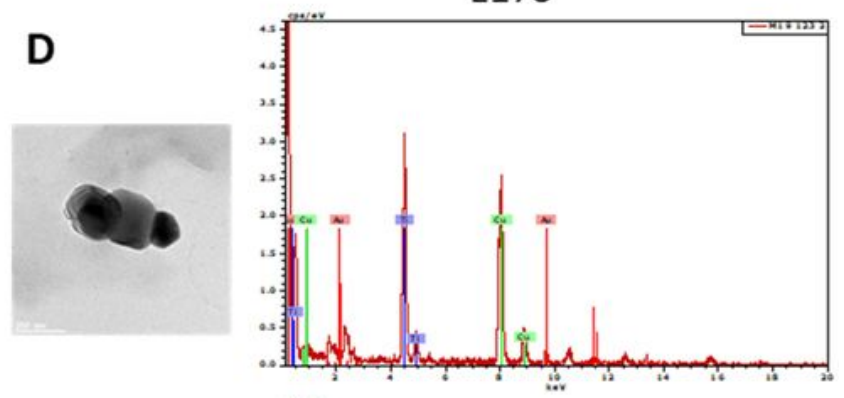

E

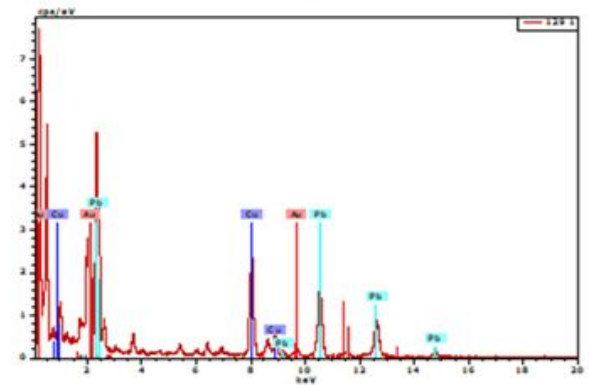

\section{Figure 3}

TEM-EDX analysis of digestive or systemic organs in mice exposed to Ref-Au or E175. TEM images of Au particles and their corresponding EDX spectra from jejunum (A), colon (B), Peyer's patches (C), liver (F) and spleen (G) of mice orally exposed to Ref-Au $(10 \mu \mathrm{g} / \mathrm{kg} \mathrm{BW} / \mathrm{d})$ for 13 weeks. TEM images of $\mathrm{Au}$ particles and their corresponding EDX spectra from the jejunum (D) and colon (E) of mice orally exposed to E175 $(10 \mu \mathrm{g} / \mathrm{kg} \mathrm{BW} / \mathrm{d})$ for 13 weeks. 


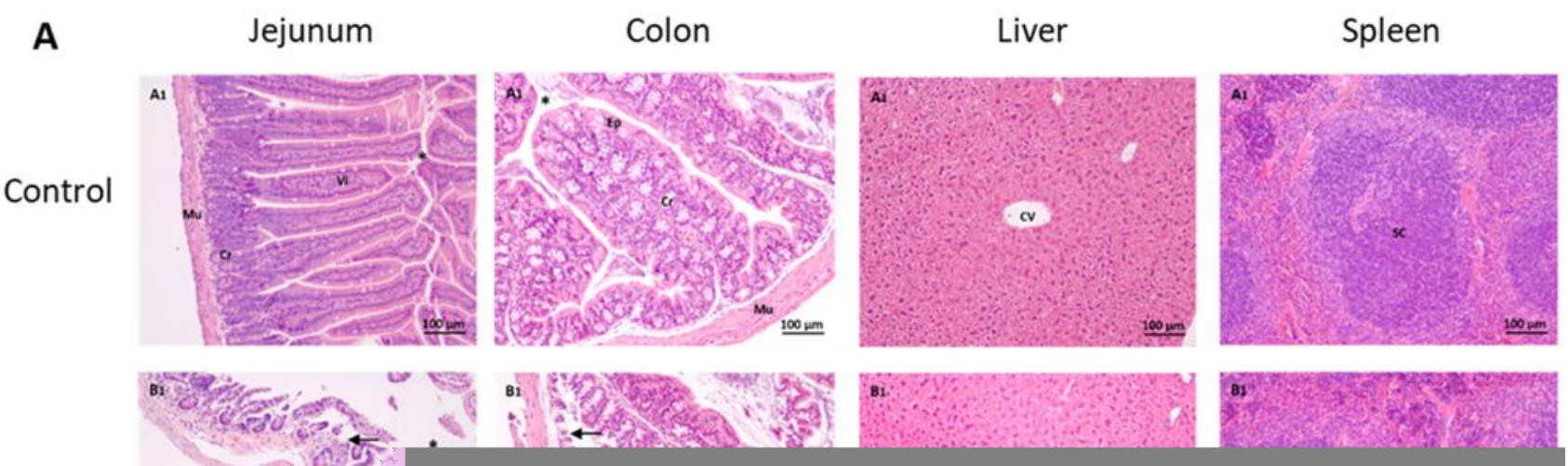

\section{Figure 4}

Histological examination of mice organs after exposure to Ref-Au or E175. (A) Haematoxylin-eosin staining of tissue sections from the jejunum, colon, liver and spleen of mice orally exposed to Ref-Au (10 $\mu \mathrm{g} / \mathrm{kg} \mathrm{BW} / \mathrm{d})$ or E175 $(10 \mu \mathrm{g} / \mathrm{kg} \mathrm{BW} / \mathrm{d})$ for 13 weeks. Arrows show inflammation of the intestinal mucosa with localized ulceration in the Ref-Au nanomaterial-treated group. In the E175-treated mice, arrowheads show erosion of the upper part of the villi. Magnification 200x. Mu: muscle; Vi: villi; Cr: crypt; Ep: 
epithelium; *: intestinal lumen; CV: central vein; SC: splenic corpuscle. (B) Histological score in the jejunum, colon, liver and spleen of mice orally exposed to Ref-Au $(10 \mu \mathrm{g} / \mathrm{kg} \mathrm{BW} / \mathrm{d})$ or E175 $(10 \mu \mathrm{g} / \mathrm{kg}$ BW/d) for 13 weeks. The data are expressed as the mean \pm SEM. ${ }^{*} \mathrm{P}<0.05$; one-way ANOVA with post hoc Tukey's test.

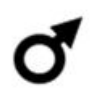

A

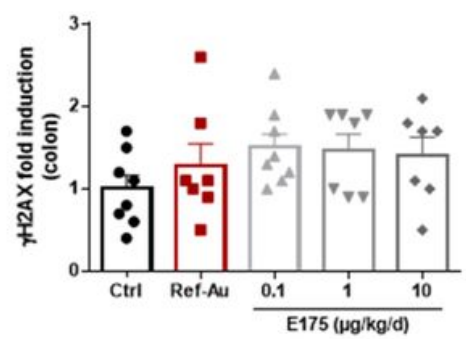

E
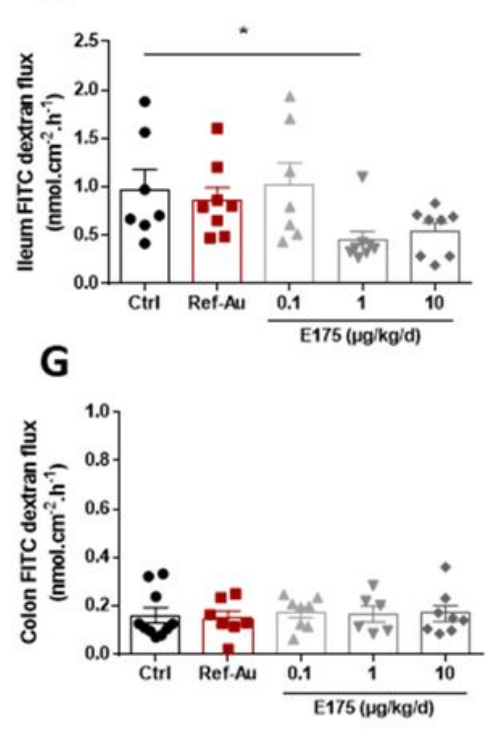

B
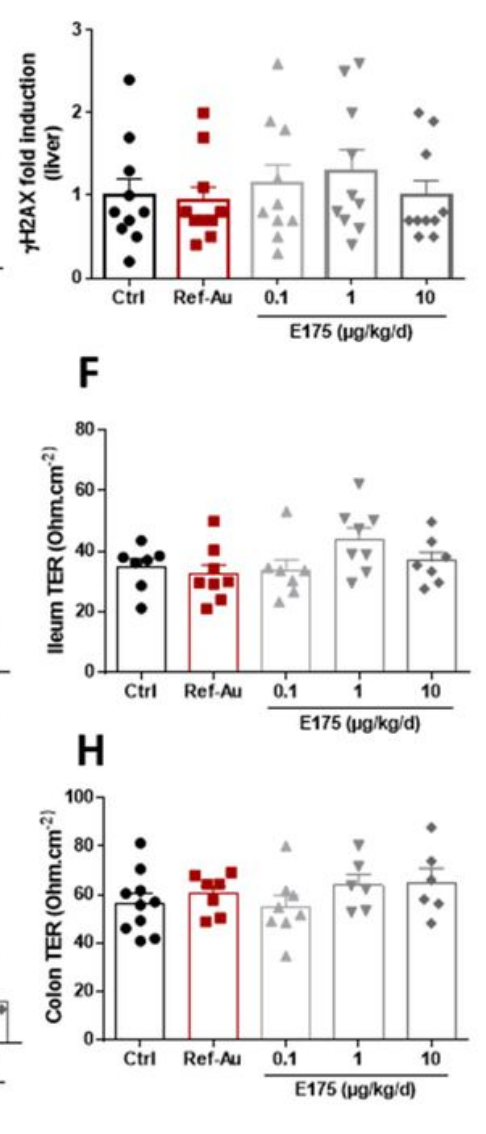

\%
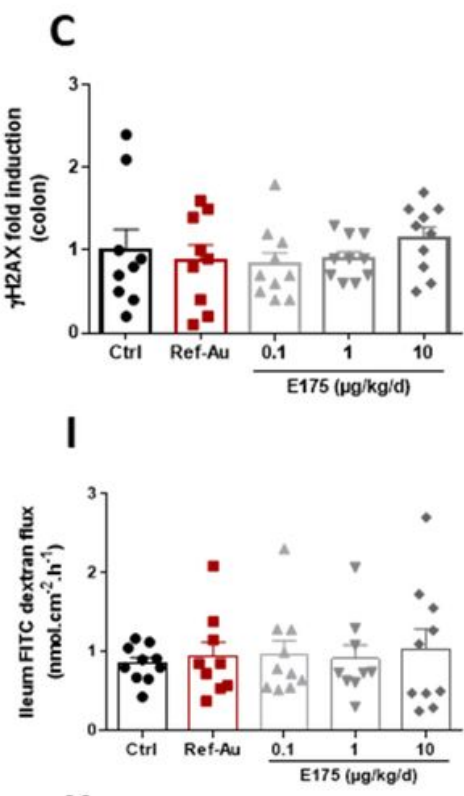

K

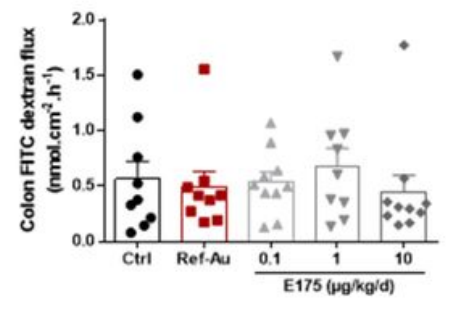

D

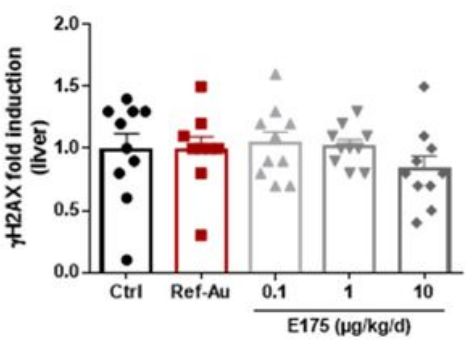

J
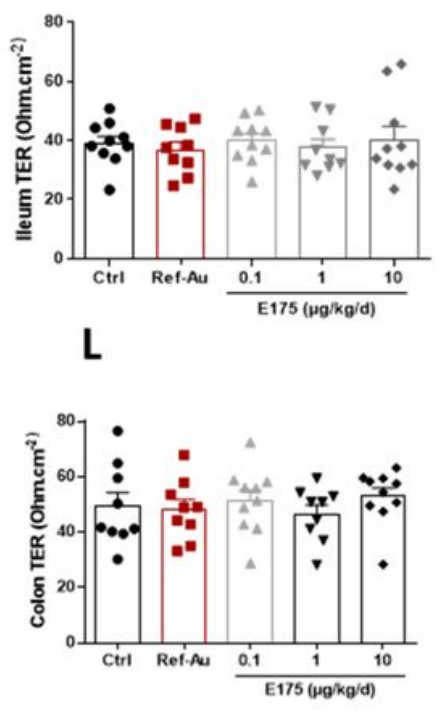

Figure 5

Genotoxicity assessment and intestinal permeability in mice exposed to Ref-Au or E175 for 13 weeks. (AD) Effect of subchronic oral treatment with Ref-Au $(10 \mu \mathrm{g} / \mathrm{kg} \mathrm{BW} / \mathrm{d})$ and E175 $(0.1,1$ and $10 \mu \mathrm{g} / \mathrm{kg} \mathrm{BW} / \mathrm{d})$ on YH2AX phosphorylation, a global genotoxic biomarker, in colon (A) and liver (B) cells of male mice and in colon (C) and liver (D) cells of female mice. Effect of subchronic oral treatment with Ref-Au $(10 \mu \mathrm{g} / \mathrm{kg}$ $\mathrm{BW} / \mathrm{d})$ and $\mathrm{E} 175(0.1 ; 1$ and $10 \mu \mathrm{g} / \mathrm{kg} \mathrm{BW} / \mathrm{d})$ on epithelial barrier integrity in the ileum $(\mathrm{E}, \mathrm{F})$ and colon (G, $\mathrm{H})$ of male mice and in the ileum $(\mathrm{I}, \mathrm{J})$ and colon $(\mathrm{K}, \mathrm{L})$ of female mice. Dextran flux and transepithelial resistance (TER) measurements for $1 \mathrm{~h}$ in Ussing chambers in ileum or colon segments from control mice (Ctrl), Ref-Au nanomaterial-treated mice (Ref-Au), and E175-treated mice. FITC dextran: Fluorescein isothiocyanate-dextran. The data are expressed as the mean $\pm \mathrm{SEM}$. ${ }^{*} \mathrm{P}<0.05$; one-way ANOVA with post hoc Tukey's test. 


\section{Figure 6}

Faecal microbiota composition in male and female mice exposed to Ref-Au or E175. Bacterial diversity based on the Shannon index in the faecal samples from male $(A)$ and female $(H)$ mice orally exposed to Ref-Au (10 $\mu \mathrm{g} / \mathrm{kg} \mathrm{BW} / \mathrm{d})$ or E175 (0.1, 1 and $10 \mu \mathrm{g} / \mathrm{kg} \mathrm{BW/d})$ for 13 weeks. Beta diversity based on BrayCurtis distance in faecal samples from male (B) and female (I) mice orally exposed to Ref-Au (10 $\mu \mathrm{g} / \mathrm{kg}$ $\mathrm{BW} / \mathrm{d})$ or E175 (0.1, 1 and $10 \mu \mathrm{g} / \mathrm{kg} \mathrm{BW} / \mathrm{d})$ for 13 weeks. Relative abundance of Bacteroidetes, Firmicutes, Proteobacteria and Actinobacteria in faecal samples from male (C-F) and female (J-M) mice orally exposed to Ref-Au (10 $\mu \mathrm{g} / \mathrm{kg} \mathrm{BW} / \mathrm{d})$ or E175 (0.1, 1 and $10 \mu \mathrm{g} / \mathrm{kg} \mathrm{BW} / \mathrm{d})$ for 13 weeks.

Firmicutes/Bacteroidetes ratio in the faecal microbiota of male $(\mathrm{G})$ and female $(\mathrm{N})$ mice orally exposed to Ref-Au $(10 \mu \mathrm{g} / \mathrm{kg} \mathrm{BW} / \mathrm{d})$ or E175 $(0.1,1$ and $10 \mu \mathrm{g} / \mathrm{kg} \mathrm{BW} / \mathrm{d})$ for 13 weeks. Relative abundance of Bifidobacterium (O) and Akkermansia muciniphila (P) in females orally exposed to Ref-Au $(10 \mu \mathrm{g} / \mathrm{kg}$ $\mathrm{BW} / \mathrm{d})$ or E175 $(0.1,1$ and $10 \mu \mathrm{g} / \mathrm{kg} \mathrm{BW} / \mathrm{d})$ for 13 weeks. For the Shannon index, differences were statistically tested using ANOVA, followed by Tukey's test. For beta diversity, PERMANOVA, $p<0.05$, followed by pairwise tests were performed. Asterisks indicate conditions that were significantly different from the controls (Ctrl). Changes in phylum relative abundances and the Firmicutes/Bacteroidetes ratio were statistically tested using ANOVA, $p<0.05$, followed by Tukey's test. The relative abundances of the genera Bifidobacterium and Akkermansia were statistically tested using the Kruskal-Wallis test, as the assumption of a normal distribution of the data was not met, $P<0.05$, followed by Dunn's post hoc test.

\section{Figure 7}

Metabolic activity of the gut microbiota in male mice exposed to Ref-Au or E175. (A-C) Orthogonal projection on latent structure-discriminant analysis (O-PLS-DA) score plots derived from ${ }^{1} \mathrm{H}$-NMR spectra of faecal extract from males exposed to E175 at 0.1 (A), 1 (B) and 10 (C) $\mu \mathrm{g} / \mathrm{kg}$ BW/day and males exposed to untreated food pellets (Ctrl). (D-F) Coefficient plots related to the O-PLS-DA models discriminating between males exposed to E175 at 0.1 (D), 1 (E) and $10(F) \mu \mathrm{g} / \mathrm{kg} \mathrm{BW/day}$ and males exposed to untreated food pellets (Ctrl). The figure shows the discriminant metabolites that are higher or lower in males exposed to E175 at 0.1 (D), 1 (E) and 10 (F) $\mu \mathrm{g} / \mathrm{kg} B W /$ day compared to males exposed to untreated food pellets. Metabolites are colour-coded according to their correlation coefficient, with red indicating a very strong positive correlation. The direction of the metabolite indicates the group with which it is positively associated, as labelled on the diagram. (G-J) The area under the curve (AUC) of the ${ }^{1} \mathrm{H}$-NMR spectra was integrated for aspartate $(\mathrm{G})$, acetate $(\mathrm{H})$, propionate $(\mathrm{I})$ and medium-chain fatty acid (MCFA) (J) signals for male mice exposed to Ref-Au $(10 \mu \mathrm{g} / \mathrm{kg} \mathrm{BW} / \mathrm{d})$ or E175 $(0.1,1$ and $10 \mu \mathrm{g} / \mathrm{kg} \mathrm{BW} / \mathrm{d})$ for 13 weeks. Each dot represents an individual mouse. Data from one independent experiment are presented as the mean \pm SEM. Statistical significance was determined by one-way ANOVA with Sidak's post hoc test; ${ }^{*} p<0.05,{ }^{* *} p<0.01,{ }^{* * *} p<0.001$. 

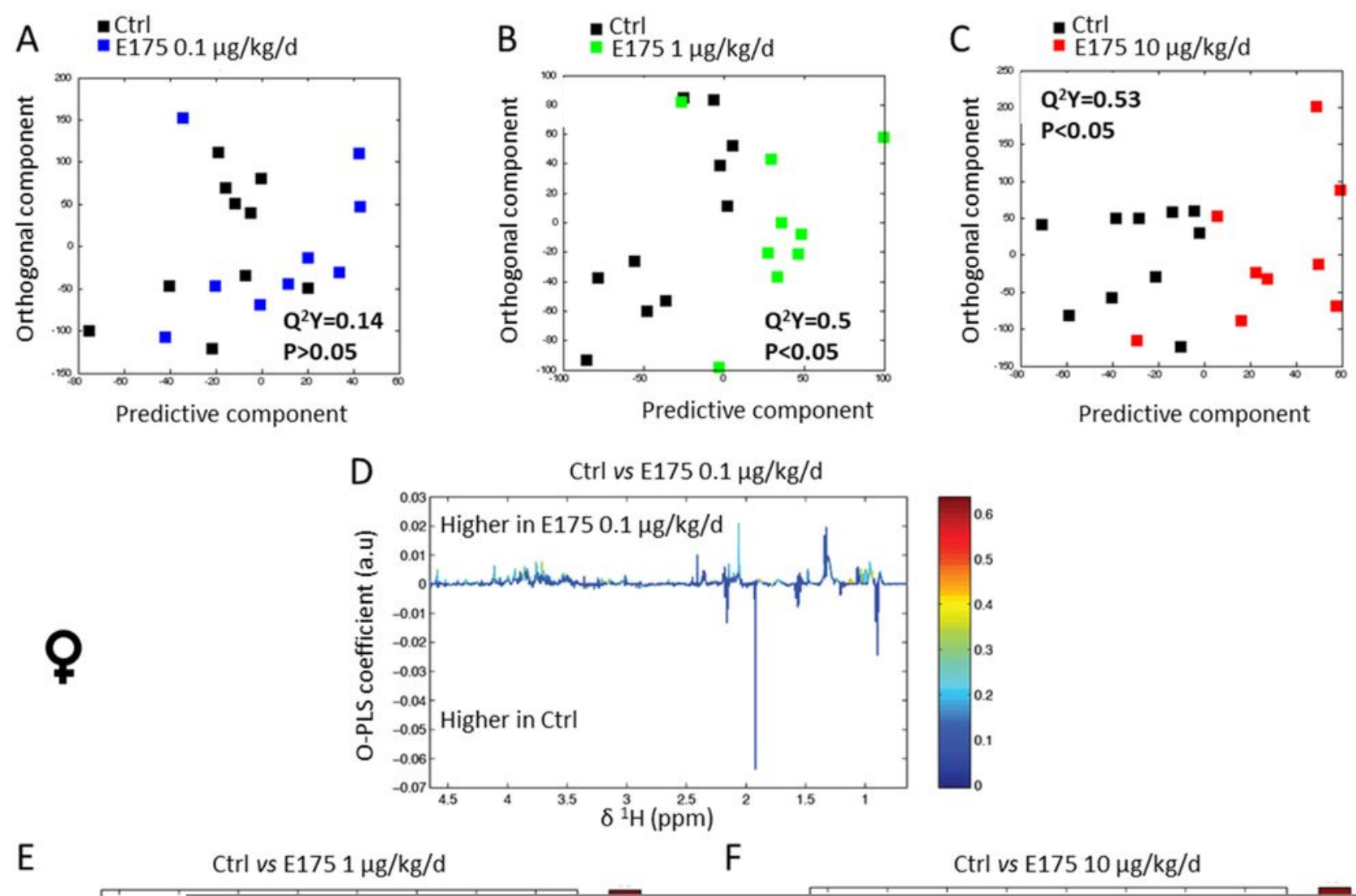

Figure 8

Metabolic activity of the gut microbiota in female mice exposed to Ref-Au or E175. (A-C) Orthogonal projection on latent structure-discriminant analysis (O-PLS-DA) score plots derived from ${ }^{1} \mathrm{H}$-NMR spectra of faecal extract from females exposed to E175 at 0.1 (A), 1 (B) and 10 (C) $\mu \mathrm{g} / \mathrm{kg} \mathrm{BW/day} \mathrm{and} \mathrm{females}$ exposed to untreated food pellets (Ctrl). (D-F) Coefficient plots related to the O-PLS-DA models discriminating between females exposed to E175 at 0.1 (D), 1 (E) and 10 (F) $\mu \mathrm{g} / \mathrm{kg} \mathrm{BW/day} \mathrm{and} \mathrm{females}$ 
exposed to untreated food pellets (Ctrl). The figure shows the discriminant metabolites that are higher or lower in females exposed to E175 at 0.1, 1 and $10 \mu \mathrm{g} / \mathrm{kg} \mathrm{BW} /$ day compared to females exposed to untreated food pellets. Metabolites are colour-coded according to their correlation coefficient, with red indicating a very strong positive correlation. The direction of the metabolite indicates the group with which it is positively associated, as labelled on the diagram. (G-I) The area under the curve (AUC) of the ${ }^{1} \mathrm{H}$-NMR spectra was integrated for acetate $(\mathrm{G})$, propionate $(\mathrm{H})$ and medium-chain fatty acid (MCFA) (I) signals for female mice exposed to Ref-Au $(10 \mu \mathrm{g} / \mathrm{kg} \mathrm{BW} / \mathrm{d})$ or E175 $(0.1,1$ and $10 \mu \mathrm{g} / \mathrm{kg} \mathrm{BW} / \mathrm{d})$ for 13 weeks. Each dot represents an individual mouse. Data from one independent experiment are presented as the mean \pm SEM. Statistical significance was determined by one-way ANOVA with Sidak's post hoc test; ${ }^{\star} \mathrm{p}<0.05,{ }^{* \star} \mathrm{p}<0.01,{ }^{* \star *} \mathrm{p}<0.001$.
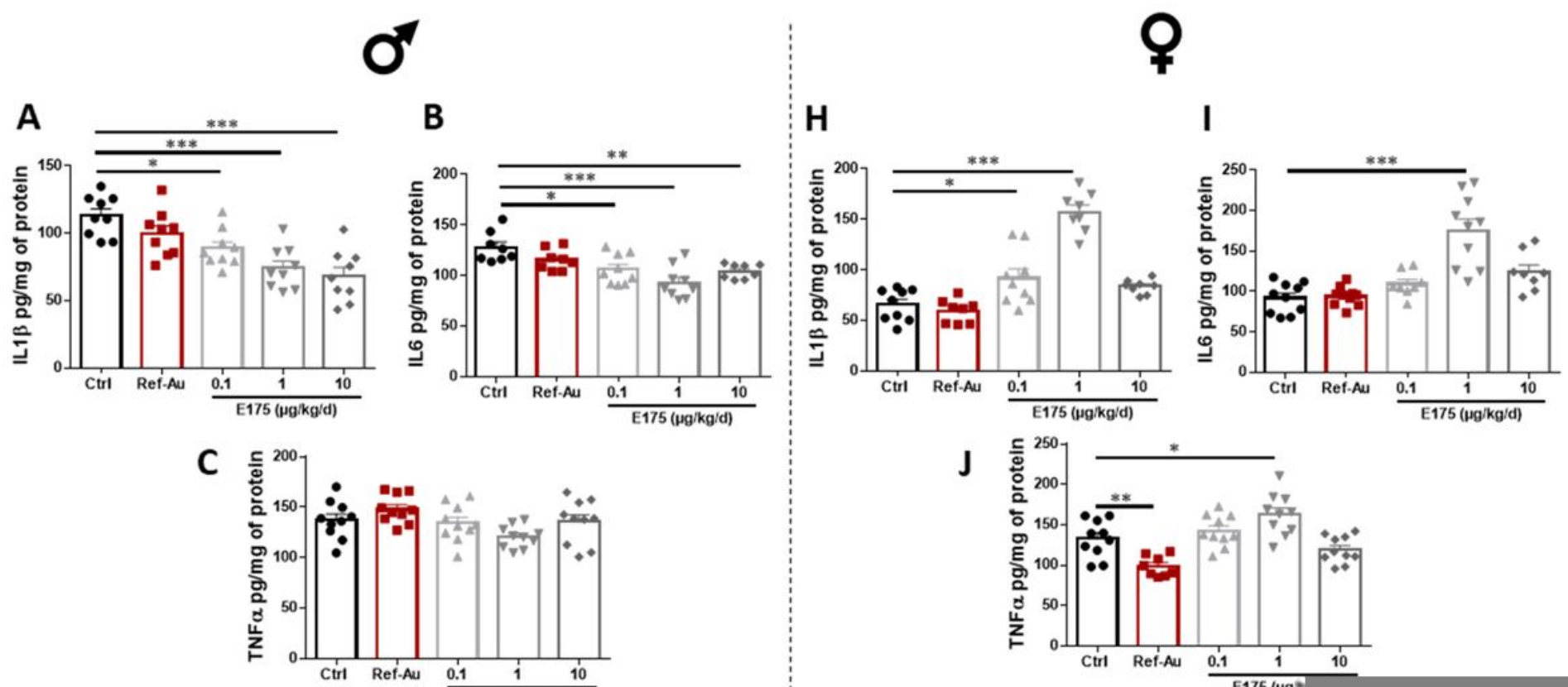

Figure 9 
Colonic immune response of male and female mice exposed to Ref-Au or E175. Protein production of the proinflammatory cytokines IL-1 $\beta(A, H)$, IL-6 (B, I) TNFa (C, J), IFNy (D, K) and IL-17 (E, L) as well as of the anti-inflammatory cytokines TGF $\beta(F, M)$ and IL-10 $(G, N)$ in the colon mucosa of male (A-G) and female $(\mathrm{H}-\mathrm{N})$ mice orally exposed to Ref-Au $(10 \mu \mathrm{g} / \mathrm{kg} \mathrm{BW} / \mathrm{d})$ or E175 $(0.1,1$ and $10 \mu \mathrm{g} / \mathrm{kg} \mathrm{BW} / \mathrm{d})$ for 13 weeks. Each dot represents an individual mouse. Data are presented as the mean \pm SEM. Statistical significance was determined by one-way ANOVA with Tukey's post hoc test or the Kruskal-Wallis test followed by Dunn's post hoc test; ${ }^{\star} p<0.05,{ }^{*} \mathrm{p}<0.01,{ }^{* \star *} \mathrm{p}<0.001$.
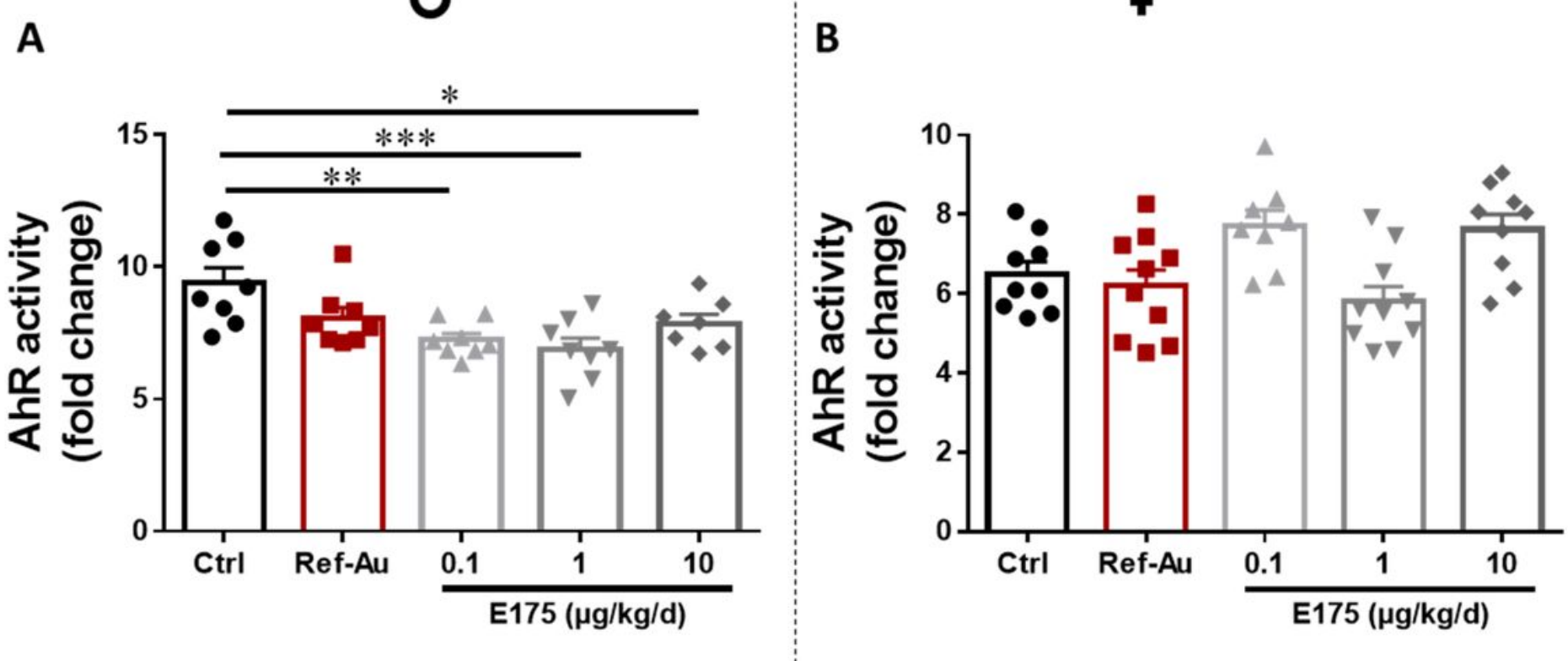

Figure 10

Capacity of faecal microbiota to activate AhR in mice exposed to Ref-Au or E175. Quantification of the faecal AhR activity of (A) male and (B) female mice exposed to Ref-Au $(10 \mu \mathrm{g} / \mathrm{kg} \mathrm{BW} / \mathrm{d})$ or E175 $(0.1,1$ and $10 \mu \mathrm{g} / \mathrm{kg} \mathrm{BW} / \mathrm{d}$ ) for 13 weeks. Data are presented as the mean \pm SEM. Statistical significance was determined by one-way ANOVA with Tukey's post hoc test; ${ }^{*} p<0.05,{ }^{* \star} p<0.01,{ }^{\star \star \star} p<0.001$.

\section{Supplementary Files}

This is a list of supplementary files associated with this preprint. Click to download.

- Supplementarydata.docx 